Mitteilungen der Österreichischen Geographischen Gesellschaft,

151. Jg. (Jahresband), Wien 2009, S. 88-118

\title{
Migration, InTEgration und StaAtsbürgerschaft IN ÖSTERREICH SEIT 1918
}

\author{
Bernhard PerchinIG, Wien*
}

\section{INHALT}

Summary .89

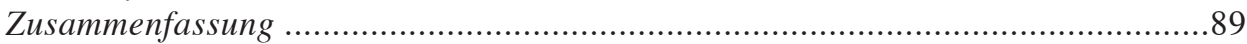

1 Zur Begrifflichkeit: internationale Migration, Binnenmigration, Staatsbürgerschaft, Integration

2 Migrationspolitik in der Ersten Republik: vom Heimatrecht zum Inlandarbeiterschutz

3 Zu Beginn der Zweiten Republik: Integration und

Ausgrenzung 1945-1960

4 Arbeitsmarktpolitik in der frühen Nachkriegszeit: der Kampf um den staatlich regulierten Arbeitsmarkt ................................................ 98

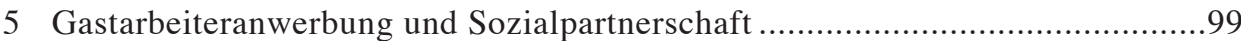

6 Die ungewollte Zuwanderung.......................................................... 101

7 Von der Sozialpartnerschaft ins Parlament..................................................... 104

8 Migrationspolitik nach dem Ende des Kalten Krieges ...................................105

9 Österreichische Migrations- und Integrationspolitik in der Europäischen Union ..................................................................... 108

10 Die Migrationspolitik der ÖVP/FPÖ-Koalitionsregierung ...........................109

11 Die Vergemeinschaftung der europäischen Migrationspolitik und ihre Auswirkungen ............................................................... 111

12 (Vorläufiger) Schluss: Migrations- und Integrationspolitik als Spiel auf mehreren Ebenen.

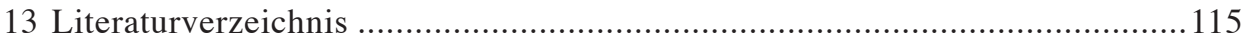

\footnotetext{
* Dr. Bernhard Perchinig, Zentrum für Europäische Integration, Donau-Universität Krems, Dr.-KarlDorrek-Straße 30, A-3500 Krems an der Donau; e-mail: bernhard.perchinig@chello.at, http://www. donau-uni.ac.at/de/department/euro/integration/index.php
} 


\section{Summary}

Migration, integration and citizenship in Austria since 1918

The article describes the history of Austrian migration, integration and citizenship policies from the beginning of the First Republic. It reveals that many political attitudes of the Second Republic had their predecessors in the First. But it also shows that by the dominance of the so-called "Social Partners" in the fields of labour market as well as migration policies a new significant element emerged and remained influential for the whole post-war period. It was not before the proceeding of European integration and Austria's membership in the European Union that it was replaced by an again more active role of both government and parliament. Regulations ruled by the European Union, however, had in the meantime taken over the command. It also reveals that the focus of discussion in this wide field has only relatively lately been shifted from the problems of the labour market to integration of migrants into the receiving society. Today, migration policies have turned into a game at several levels: the levels of the European Union, of its member states, of provinces and communes as well as of "Social Partners", who have, however, essentially lost in importance. Under these conditions radical changes of paradigms have become unlikely and gradual modifications of policies are favoured. It is therefore expected that in the longer run Austrian migration and integration policies, like in other EU member states, will try to be directed towards a combination of both a rather restrictive control over new migration influx and more openness for cultural diversity. It has also become more likely that it will develop into a rather consensual field of politics.

\section{Zusammenfassung}

Der Artikel beschreibt die Geschichte des österreichischen Migrations-, Integrations- und Staatsbürgerschaftspolitik seit dem Beginn der Ersten Republik. Es wird dabei deutlich, dass viele Politikmuster der Zweiten Republik im Österreich der Zwischenkriegszeit ihre Vorläufer hatten. Doch zeigt sich auch, dass mit der Dominanz der Sozialpartner im Bereich der Arbeitsmarkt- und damit auch der Migrationspolitik ein neues Element markant in Erscheinung trat und die gesamte Nachkriegszeit bestimmend blieb. Es wurde erst mit dem Voranschreiten des europäischen Integrationsprozesses und durch die Mitgliedschaft Österreichs in der Europäischen Union von einer wieder stärkeren Aktivität der Regierung und des Parlaments abgelöst, wobei jedoch zunehmend Regelungen der Europäischen Union nationales Recht vorbestimmten. Außerdem wird deutlich, dass sich der Schwerpunkt der Diskussion in diesem weiten Themenfeld erst relativ spät von einer Fokussierung auf die Problematik des Arbeitsmarktes auf eine Fokussierung auf das Problem der Integration von Migranten in die Gesellschaft des Empfängerlandes verlagerte. Heute ist Migrationspolitik zu einem Spiel auf mehreren Ebenen geworden: auf jener der Europäischen Union, der Mitgliedsstaaten, der Bundesländer und Gemeinden und der Sozialpartner, die jedoch wesentlich an Bedeutung verloren haben. Solche Verhältnisse machen radikale Paradigmenwechsel 
unwahrscheinlich und favorisieren schrittweise Politikentwicklung. Es wird daher auf mittlere Sicht erwartet, dass sich die Migrations- und Integrationspolitik auch in Österreich, ähnlich wie in anderen EU-Staaten, an einer Kombination aus restriktiver Steuerung der Neuzuwanderung und größerer Offenheit gegenüber kultureller Vielfalt orientieren wird, und dass sie sich zu einem, wenn schon nicht konsensualen, so doch weniger umstrittenen Politikfeld entwickelt.

\section{Zur Begrifflichkeit: internationale Migration, Binnen- migration, Staatsbürgerschaft, Integration}

\subsection{Internationale Migration}

Die Vereinten Nationen (UNO) definieren einen internationalen Migranten als eine Person, die ihren Ort des gewöhnlichen Aufenthalts - verstanden als jener Ort, wo sie tägliche oder wöchentliche Ruhe- und Freizeit verbringt - verlässt und sich an einem anderen Ort niederlässt, sodass dieser der neue Ort des gewöhnlichen Aufenthalts wird (United Nations 1998, S. 18). Geschieht dies für länger als ein Jahr, so spricht die UNO-Definition von einem Langzeitmigranten. Allerdings zählt kaum ein Land der Welt Kurzzeitmigranten oder registriert den ,gewöhnlichen Aufenthalt“. In der Praxis stehen höchstens Daten über den Geburtsort oder über die Staatsangehörigkeit zur Verfügung.

Bezogen auf ihre Definition gab es laut Schätzungen der Vereinten Nationen im Jahr 2005 weltweit etwa 195 Mio. Migranten, was etwa 3,3\% der Weltbevölkerung entsprach. Die Zahl der internationalen Migranten stieg in den letzten Jahrzehnten auch deutlich an - von 155 Mio. im Jahr 1990 auf eben 195 Mio. im Jahr 2005 (UNITED Nations 2008). Dennoch: internationale Migration ist eine Erfahrung für eine Minderheit der auf der Erde lebenden Menschen; die meisten verbringen ihr ganzes Leben in ihrem Geburtsland.

\subsection{Binnenmigration}

Häufiger, doch von den Vereinten Nationen nicht erfasst und daher nur über Schätzungen zugänglich, ist Binnenmigration. Allein in China, so die Schätzung, wechselten im Jahr 2008 mehr als 140 Mio. Menschen ihren Wohnsitz oder zogen vom Land in die Stadt (Council on Foreign Relations 2009). In Europa und der „westlichen“ Welt wird Binnenmigration selten als soziales oder politisches Thema diskutiert. Dies hat vor allem damit zu tun, dass die europäischen Nationalstaaten spätestens seit dem Zweiten Weltkrieg als gesellschaftliche und ökonomische „Container“ eine weitgehende Homogenisierung der Schulen und Ausbildungszertifikate, Arbeitsmärkte und Rechtsräume erreicht haben. Der Wechsel von einem österreichischen Bundesland in ein anderes oder vom Land in die Stadt kann zwar den Einzelnen durchaus noch im- 
mer mit einer teilweise deutlich unterschiedlichen Alltagskultur konfrontieren, doch hat er/sie die gleiche Rechtsstellung wie die Altansässigen, die Umgangssprache ist zumeist die Gleiche, und Ausbildungsabschlüsse und Berufserfahrung werden am Arbeitsmarkt anerkannt.

\subsection{Staatsbürgerschaft}

In einer Welt von Nationalstaaten ist die Staatsbürgerschaft ein für das Leben zentraler Rechtsstatus, den Hannah ArEndT treffend als Realisierung des grundlegenden Menschenrechts - „des Rechts, Rechte zu haben“ verstanden hat (ARENDT 2007, S. 612ff.). Thomas Marshall hat anhand der Entwicklung des britischen Citizenship-Regimes die Entwicklung von Staatsbürgerschaft weg von einem Autoritätsverhältnis hin zu einem Bündel von zivilen, politischen und sozialen Rechten des Bürgers gegenüber dem Staat beschrieben und damit ein Modell für das Verständnis des grundlegenden Wandels des Verhältnisses von Bürger und Staat nach dem Zweiten Weltkrieg vorgelegt (Marshall 1992). Spätere Studien haben darauf hingewiesen, dass dieses Rechtsbündel inzwischen in weiten Bereichen aus der Staatsbürgerschaft in die Menschenrechte verschoben wurde (vgl. u.a. HAMmar 1990; BAubÖск 1994; Sosysal 1994). Wesentlich sind jedoch bis heute die mit der Staatsbürgerschaft verbundenen aufenthalts- und einreisebezogenen Rechte: das uneingeschränkte Aufenthaltsrecht, das Recht auf freie Wahl des Aufenthaltsorts im Staatsterritorium sowie das uneingeschränkte Recht auf Rückkehr in dieses. Letzteres ist ein im Migrationskontext besonders relevantes Recht, ermöglicht es doch die temporäre Migration mit der Möglichkeit der Rückkehr in einen „,sicheren Hafen“. Auch das mit der Staatsbürgerschaft verbundene Recht auf einen Reisepass, dessen „Qualität“1) die Stellung des jeweiligen Staates in der Hierarchie der Staatenwelt spiegelt, ist hier zu erwähnen (TORPEY 2000).

Bis zur Einführung der Bürgerschaft der Europäischen Union (EU) sicherte in Österreich nur die Staatsbürgerschaft das Recht auf Gleichbehandlung am Arbeitsmarkt und im Bildungs-, Gesundheits- und Sozialbereich sowie das Wahlrecht auf allen Ebenen. Diese Rechte wurden seit den 1960er Jahren in den meisten Einwanderungsländern Mittel- und Westeuropas, wenn auch in deutlich unterschiedlichem Ausmaß, von der Staatsbürgerschaft entkoppelt und vermehrt an die Aufenthaltsdauer in einem Staat geknüpft, wodurch zwischen Fremden und Staatsbürgern ein Zwischenstatus des „Denizens“ (Hammar 1990), des „Wohnbürgers“ (B AuвÖск 2001, S. 20) entstand.

Das Institut der Unionsbürgerschaft, das in einem EU-Staat lebenden Staatsangehörigen anderer EU-Staaten eine den eigenen Staatsbürgern ähnliche Rechtsstellung gewährt, ist ein Beispiel für diesen „Denizenship“-Status: Unionsbürger haben ein weitgehend sicheres, wenn auch nicht unbeschränktes Aufenthalts- und Wiedereinreiserecht. Mit Ausnahme hoheitlicher Aufgaben verfügen sie über freien Arbeitsmarktzugang und das Recht auf Gleichbehandlung im Bildungs-, Gesundheits- und Sozialbereich. Ihr Wahlrecht ist allerdings auf die kommunale Ebene begrenzt.

\footnotetext{
1) Messbar an der Zahl der Länder, in die (visafrei) eingereist werden kann.
} 
Man wollte mit der Unionsbürgerschaft in erster Linie einen freien Arbeitsmarkt mit einem freizügigen Migrationsregime schaffen. Sie sollte verhindern, dass der freie Zugang zum Arbeitsmarkt durch staatliche Interventionen behindert wird. Die Freizügigkeit war zunächst auch auf ökonomisch aktive Personen - Arbeitnehmer, Selbständige und Unternehmer - beschränkt.

Im Zuge des europäischen Einigungsprozesses entstand so ein Migrationsregime, das sich an der Bewegungsfreiheit innerhalb von Staaten orientierte. Es räumte Staatsangehörigen anderer Mitgliedsstaaten sukzessiv mehr Rechte ein, um Mobilitätshindernisse zu beseitigen. Mit der späteren Ausdehnung der Rechte auf alle Staatsangehörigen der Mitgliedsstaaten (nicht nur auf ökonomisch Aktive) entstand ein europäischer Migrationsraum, der dem Einzelnen die freie Migrationsentscheidung ermöglicht, weil rechtliche Unterschiede nicht mehr bestehen. Davon ausgenommen sind bislang nur die seit 2004 beigetretenen Mitgliedstaaten, deren Staatsbürgern die freie Migration in die EU-15 erst nach einer Übergangsfrist gewährt wird.

Von der gleichen Grundidee getragen ist der Status eines „langansässigen Drittstaatsangehörigen“. Er stellt Nicht-EU-Bürger vor allem beim Arbeitsmarktzugang, beim Zugang zum Bildungs-, Gesundheits- und Sozialsystem sowie zu Gütern und Dienstleistungen weitgehend mit Unionsbürgern gleich, gewährt ihnen aber keinen so sicheren Aufenthaltsstatus und keine politischen Mitbestimmungsrechte.

\subsection{Integration}

Integration ist ein multifaktorieller Prozess, der durch eine rechtlich starke Position sicher erleichtert wird. Doch ist diese nicht immer eine notwendige und nie eine ausreichende Bedingung. Zudem, und dies macht die Sache noch komplexer, unterliegt der Integrationsbegriff einem historischen Wandel. Die Kriterien, die heute angelegt werden, standen vor 20 Jahren noch nicht zur Diskussion, und es ist zu erwarten, dass sich der Begriff weiter wandelt. Wer die Geschichte der Migrations- und Integrationspolitik darstellt, sollte daher den Diskurs immer historisch verorten und sich über die Historizität der Analysekategorien im Klaren sein.

Dieser Beitrag versucht eine Rekonstruktion der österreichischen Migrations-, Staatsbürgerschafts- und Integrationspolitik seit 1918. Unter Migrationspolitik wird die Politik zur Regelung der Zu- und Abwanderung aus dem Ausland verstanden; unter Staatsbürgerschaftspolitik die Regulierung des Staatsbürgerschaftserwerbs (und -verlusts). Mit Integrationspolitik sind alle politischen Maßnahmen gemeint, welche die Rechtsstellung von Migranten im Zielland betreffen und deren Teilhabe an der Gesellschaft des Ziellandes beeinflussen möchten. 


\section{Migrationspolitik in der Ersten Republik: vom Heimatrecht zum Inlandarbeiterschutz}

Sowohl Ein- und Auswanderung als auch die Wanderung innerhalb des Staatsgebietes unterlagen in der Österreichisch-Ungarischen Monarchie kaum politischer Gestaltung durch den Staat. Das wichtigste Steuerungsinstrument der Migration war das bei den Gemeinden angesiedelte und durch Geburt oder Eheschließung erworbene Heimatrecht. Es verpflichtete die Gemeinden, verarmte Gemeindebürger zu unterstützen. Es berechtigte sie aber auch, in der Gemeinde Wohnhafte, die nicht das Heimatrecht besaßen, in ihre Geburtsgemeinde abzuschieben. Diese Form der Migrationskontrolle diente also nicht der Steuerung der Arbeitsmigration, sondern der Armenabwehr.

Auch die Auswanderung ins Ausland blieb trotz mehrerer Anläufe zu einem Auswanderungsgesetz bis zum Ende der Monarchie unreguliert. Einige Vorarlberger Gemeinden verknüpften sogar das Heimatrecht mit der Auswanderungsfreiheit, indem sie unerwünschte Einwohner - Bettler, Kranke, Kriminelle - davon zu überzeugen suchten, auszuwandern oder sogar deren Auswanderung selbst organisierten (SENSENIG 1998, S. 171ff.).

In der Ersten Republik kam es erstmals zu einer Einschränkung des freien Arbeitsmarktzuganges. Nachdem im Jahr 1921 mit nur 1,2\% Arbeitslosigkeit praktisch Vollbeschäftigung erreicht worden war (SENSENIG 1998, S. 372), kam es zu massiver Arbeitskräfteknappheit in Gewerbe, Industrie und Landwirtschaft. Landwirtschafts-, Innen- und Sozialministerium förderten daraufhin die Einwanderung durch eine liberale Handhabung der Bestimmungen zu Identitätsdokumenten und Einreisepapieren. Angeworben wurde vor allem in den Ländern Deutschland, Italien und Tschechoslowakei, mit denen auch entsprechende Anwerbeabkommen geschlossen wurden. Da die Angeworbenen kein Vertrauen in die österreichische Währung hatten, wurden sie zumeist in ihrer Landeswährung bezahlt. In Vorarlberg bezahlte die Stickereiindustrie auch die österreichischen Arbeiter in Schweizer Franken, um deren Abwanderung in die Schweiz zu verhindern (SEnsenig 1998, S. 373).

Erste Kritik an der Förderung der Arbeitseinwanderung kam Anfang der 1920er Jahre von den Gewerkschaften, den Arbeiterkammern sowie den Ländern und Gemeinden. Während Letztere vor allem auf die Schwierigkeiten hinwiesen, genügend Devisen für die Bezahlung der ausländischen Arbeiter zu bekommen, monierten Erstere den Stopp der Zuwanderung ,fremdsprachiger Arbeiter“. So forderte die Arbeiterkammer Salzburg im Jahr 1921 von der Landesregierung, „geeignete Maßnahmen zu treffen, damit die einheimische arbeitende Bevölkerung gegen die drohende Überflutung fremdsprachiger Arbeiter entsprechend und wirksam geschützt ist" (zitiert nach SENSENIG 1998, S. 377). Auch die Wiener Arbeiterkammer vertrat diese Position. Hintergrund war der massive Protest der Bauarbeitergewerkschaft gegen die Anwerbung italienischer Bauarbeiter durch die Wiener Unternehmen. Das Sozialministerium übernahm Anfang 1922 die Position der Arbeiterkammern und forderte das Innenministerium auf, „,die Zuwanderung fremdländischer Arbeiter nach Möglichkeit hintanzustellen“ (zitiert nach SENSENIG 1998, S. 379). Ausgenommen von den Beschränkungen blieben 
aus außenpolitischen Gründen reichsdeutsche Arbeitnehmer. Der Arbeitsmarkt wurde also als ein nationales Gut definiert.

Als mit dem Beginn der Wirtschaftskrise im Jahr 1923 zunehmend deutsche Arbeitslose in Österreich Arbeit suchten und österreichischen Bewerbern vorgezogen wurden, reagierte die Bundesregierung auf Druck der Gewerkschaften und Arbeiterkammern mit dem Inlandarbeiterschutzgesetz (SEnsenig 1998, S. 399ff.). Dieses „Bundesgesetz vom 19. Dezember 1925 über die zeitweilige Beschränkung der Beschäftigung ausländischer Arbeiter und Angestellter“ erlaubte die Neuanwerbung von Ausländern in der Regel nur dann, wenn sie Fachkenntnisse mitbrachten, die für den Erhalt von inländischen Arbeitsplätzen notwendig waren. Es gab den Behörden die Möglichkeit, illegale Beschäftigung zu bekämpfen. Die Bevorzugung der Reichsdeutschen war damit aufgehoben, alle Ausländer unterlagen nun den gleichen Bestimmungen. Zwar blieb das staatliche Wanderungsamt als letzte Instanz für die Erteilung von Genehmigungen verantwortlich. Es konnte jedoch seine Entscheidungsbefugnisse auf die „Industriellen Bezirkskommissionen“ in den Bundesländern übertragen. Diese bald nach Kriegsende gegründeten Kommissionen beteiligten Arbeitgeber- und Arbeitnehmervertreter bei der Entscheidungsfindung (SENSENIG 1998, S. 400).

Damit finden sich bereits in der Ersten Republik zwei auch für die Zweite Republik lange Zeit prägende Elemente der Migrationspolitik: (1) der „Inländerprimat“ - die Bevorzugung österreichischer Arbeitnehmer gegenüber Ausländern nach dem Motto "last hired, first fired” - und die Definition von Ausländerbeschäftigung als tendenziell unerwünschte „Ausnahme von der Regel“; (2) die paritätische Einbindung von Arbeitgeber- und Arbeitnehmervertretern in die Steuerung der Ausländerbeschäftigung außerhalb der parlamentarischen Arena.

\section{Zu Beginn der Zweiten Republik: Integration und Ausgrenzung 1945-1960'}

Die meisten Darstellungen der Migrationsgeschichte der Zweiten Republik datieren den Beginn der Einwanderung auf die 1960er Jahre (so Fassmann \& MünZ 1995). Damit wird eine quantitativ bedeutende Migrations- und Integrationsphase der Nachkriegsjahre, nämlich die Integration der „Volksdeutschen“ und der Flüchtlinge zwischen dem Kriegsende und dem Beginn der 1960er Jahre ausgeblendet. „Der Anteil dieser integrierten Flüchtlinge - zumeist, aber keineswegs ausschließlich deutschsprachige Vertriebene oder Aussiedler aus Osteuropa - belief sich in Österreich auf 6 bis $8 \%$ der Gesamtbevölkerung des Landes. In der Bundesrepublik Deutschland erreichte der Anteil der nach dem Zweiten Weltkrieg dauerhaft integrierten Flüchtlinge sogar 15\% der Bevölkerung“ (BAumgartner 2009, S. 21). Im letzten Kriegsjahr und nach dem Ende des Zweiten Weltkrieges gab es in Europa etwa 30 Mio. Flüchtlinge und Vertriebene (Volf 1995, S. 418).

\footnotetext{
2) Teile dieses sowie der folgenden Kapitel beruhen auf Perchinig 2009.
} 
In Österreich waren zwei große Gruppen bestimmend: erstens die "Displaced Persons" (DPs), vom NS-Regime zwangsrekrutierte Arbeiter, Kriegsgefangene und jüdische Überlebende der Konzentrationslager; und zweitens die aus Ost- und Mitteleuropa vertriebenen deutschen Minderheiten, die sogenannten „Volksdeutschen“. Beide Gruppen zusammen beziffert die Literatur auf 1,4 bis 1,65 Mio. Menschen (Volf 1995, S. 420) - immerhin zwischen einem Fünftel und einem knappen Viertel der damaligen Bevölkerung des Landes. Bis zum 30. Juni 1947 wurden etwa 900.000 Menschen mit Hilfe der alliierten Hilfsorganisation UNRRA (United Nations Relief and Rehabilitation Administration) - teilweise auch gegen ihren Widerstand - in ihre Herkunftsländer zurückgebracht.

Die Differenzierung zwischen “Displaced Persons” und „Volksdeutschen“ ist direkt auf die Kriegsgeschichte zurückzuführen: aufgrund des Potsdamer Abkommens wurden die Deutschsprachigen der mittel- und osteuropäischen Staaten verallgemeinernd als Kollaborateure des Nazi-Regimes angesehen und ihre „Rückführung“ nach Deutschland beschlossen. Gut eine Million wurde nach dem Kriegsende nach Österreich vertrieben und war hier vom Zugang zu den Hilfsmitteln der alliierten UNRRA und später der IRO (International Refugee Organisation) ausgeschlossen.

Da Deutschland versuchte, die Zahl der aufgenommenen Volksdeutschen möglichst niedrig zu halten, blieben mehr Menschen länger in Österreich als die UNRRA Kapazitäten hatte. Österreich wollte aber auch keinen Konflikt mit der Sowjetunion, die massiv Widerstand gegen die Unterstützung der deutschsprachigen Bevölkerung leistete, und verunmöglichte ihnen auch deshalb den Zugang zu Hilfsleistungen.

Erst mit der Etablierung des Mandats des UNHCR (United Nations High Commissioner for Refugees) im November 1951 erhielten auch die deutschsprachigen Flüchtlinge Zugang zu Hilfsleistungen (Volf 1995, S. 422). Viele Volksdeutsche waren bis dahin gezwungen, privat bei Verwandten oder Bekannten unterzukommen und sich durch verschiedene Arbeiten, vor allem in der Landwirtschaft oder am Schwarzmarkt, über Wasser zu halten. Dadurch kamen sie jedoch auch frühzeitig in Kontakt mit Arbeitgebern, und es entstanden dichte Netzwerke zur einheimischen Bevölkerung.

Im Gegensatz zu den Volksdeutschen hatten Displaced Persons Zugang zu den Hilfeleistungen der UNRRA. Sie lebten zumeist in Flüchtlingslagern, die von der UNRRA verwaltet wurden. Zu den Displaced Persons der ersten Nachkriegszeit kamen zwischen 1945 und 1947 mehrere Wellen jüdischer Flüchtlinge vor den Pogromen in Osteuropa; die größte nach dem Pogrom von Kielce (Polen) im Sommer 1947. Österreich zeigte kein Interesse an einer Ansiedlung. Für Österreich wäre dies einem Schuldeingeständnis und dem Anerkennen einer Pflicht zur Wiedergutmachtung nach dem Holocaust gleichgekommen. Aber auch der neu gegründete Staat Israel drängte auf eine rasche Weiterreise (BAuböck \& Perchinig 2006, S. 728).

Es gab etwa 53 gesonderte Lager für die insgesamt etwa 200.000 bis 225.000 jüdischen Flüchtlinge und geschätzte weitere 200 Lager für andere DPs. Insgesamt lebten zwischen 1945 und 1954 etwa 1,74 Mio. Flüchtlinge zumindest zeitweise in diesen Lagern, mehr als eine halbe Million blieb auf Dauer in Österreich (BAUMGARTNER 2009, S. 26). Diese Lager - manche hatten die Bevölkerungszahl von mittleren Städten - waren oft Anlass für soziale und wirtschaftliche Konflikte. Die DPs unterlagen strengen Regeln in Bezug auf den Zugang zum Arbeitsmarkt und mussten sich bis 
zu ihrer Einbürgerung oder Auswanderung jährlich Arbeitsgenehmigungen besorgen. Sie hatten daher nur beschränkte Beschäftigungsmöglichkeiten. Diese Einschränkung schrieb Österreich sogar anlässlich der Unterzeichnung der Genfer Flüchtlingskonvention in einem Vorbehalt fest.

In den Lagern wurden die DPs jedoch von der UNRRA entsprechend den internationalen Verpflegungssätzen verpflegt und waren dadurch oft besser mit Nahrungsmitteln versorgt als österreichische Staatsbürger. In der Öffentlichkeit entstand so schnell das Bild der auf Kosten der Bevölkerung lebenden „Schmarotzer“, während die Volksdeutschen als fleißig und arbeitsam wahrgenommen wurden. Typisch für diese, an manch heutige Asyldebatte gemahnende Wahrnehmung ist die von BAUMGarTnER zitierte Wortmeldung des Abgeordneten zum Nationalrat der Sozialistischen Partei Österreichs ${ }^{3)}$ (SPÖ) Felix STIKA aus dem Jahr 1946:

„Diese Ausländer werden von der UNRRA verpflegt, und zwar zu den internationalen Verpflegssätzen. Sie erhalten daher 2.000 bis 2.500 Kalorien, während sich der österreichische Staatsbürger selber mit 900 und höchstens 1.150 Kalorien zufrieden geben muß. Wir haben ein solches Lager in Tirol, das allein 38.000 Polen und Ukrainer umfaßt. Wir haben ein ähnliches Lager, ein berïchtigtes Lager, in St. Johann im Pongau in Salzburg. Dort hausen 1.500 Jugoslawen, Offiziere und Soldaten. Der Kommandant dieses Lagers ist ein jugoslawischer General namens Petrovic. Sie alle stehen unter dem Schutz der amerikanischen Militärregierung, sie haben keine Verpflichtung zu arbeiten, im Gegenteil (Zwischenrufe), wer arbeitet, der muß sich seine Verpflegung bezahlen, wer aber nicht arbeitet, der bekommt sie umsonst! Diese Zustände müssen wir einmal feststellen. Daß unter diesen Umständen der Schwarz- und Schleichhandel blühen, das ist eine selbstverständliche Tatsache. (....) Diese Schieber- und Schleichhändlerlager müssen wir außerdem noch betreuen und dafür ungeheure Geldsummen ausgeben ...(...)... Diese ungeheure Masse von untätigen Ausländern ist eine schwere Belastung nicht nur innenpolitisch, sondern auch außenpolitisch. Wir können und wir werden sie auf die Dauer in Österreich nicht ertragen können. Diese Menschen gehören in ihre Heimat zurückgeführt, oder um mich drastischer auszudrücken, sie gehören einfach abgeschoben (Zwischenruf: Die Besatzungsbehörden nehmen sie ja nicht auf!), weil sie uns nur Schwierigkeiten bereiten." (Aus dem stenographischen Protokoll der Sitzung des Österreichischen Nationalrates vom 23.5.1946, zitiert nach Baumgartner 2009, S. 27.)

Nicht zuletzt deshalb, weil die Alliierten die Kosten der Flüchtlingsbetreuung der Republik Österreich verrechneten, bildete sich unter den österreichischen Parteien ein Konsens darüber, die Lager der DPs möglichst schnell zu schließen, ihre Insassen außer Landes zu bringen und Integration zu verunmöglichen. Auch die Alliierten teilten diese Haltung im Wesentlichen (Volf 1995, S. 419).

Auch gegenüber den Volksdeutschen verhielt sich die Politik zuerst ablehnend. Ihre Versorgung wurde als Belastung dargestellt und Österreich machte sich bei den Alliierten für ihre Abschiebung stark. Ausgenommen sein sollten nur jene, die in der Monarchie die österreichische Staatsbürgerschaft besessen hatten und keine Ansprüche

\footnotetext{
3) Heute Sozialdemokratische Partei Österreichs.
} 
auf staatliche Unterstützung stellten. Die Sudetendeutschen bevorzugte man deshalb, weil sie der „österreichischen Bevölkerung ethnografisch nahe verwandt sind.“ Alle anderen Volksdeutschen sollten nur dann bleiben können, wenn „die österreichische Wirtschaft [an ihrer Arbeitskraft] interessiert ist“ (KARNER \& STANGLER 2005, S. 46).

Als in den ersten Nachkriegsjahren der Arbeitskräftemangel in Landwirtschaft und Gewerbe deutlich spürbar wurde, änderte sich die Position der Regierung. Nun wollte man sich, so Bundeskanzler FIGL in seiner Regierungserklärung vom 9.11.1949, für die in Österreich aufhältigen „Heimatvertriebenen“ - also die Volksdeutschen - einsetzen (Volf 1995, S. 420).

Ein wichtiger Grund für den Meinungsumschwung war auch die gute politische Organisation der Volksdeutschen, die sowohl in der Österreichischen Volkspartei (ÖVP) als auch in der SPÖ aktiv waren und ihre Interessen geltend machten. Im Jahr 1950 beschloss der Ministerrat die Einsetzung eines Ministerkomitees für die Behandlung der Flüchtlingsfragen der Volksdeutschen, dem ein Beirat für Flüchtlingsfragen zur Seite gestellt wurde. Dieser wurde von den in den Parlamentsparteien etablierten Vertretern der Volksdeutschen beschickt und arbeitete einen Integrationsplan aus.

Aufgrund der Vorschläge des Beirats, aber auch wegen der beginnenden Abwanderung von Volksdeutschen nach Deutschland, kam es zu ihrer forcierten Integration: Im Jahr 1952 wurden sie von der für die DPs geltenden Pflicht der Beschaffung einer Arbeitsgenehmigung befreit und es wurde ihnen freier Arbeitsmarktzugang gewährt. 1954 erhielten sie schließlich die Möglichkeit, die österreichische Staatsbürgerschaft nach einer Loyalitätserklärung per einfacher Deklaration zu minimalen Kosten zu erwerben (STIEBER 1995).

Für die DPs und andere Flüchtlinge gab es keine vergleichbaren Integrationsangebote. Viele versuchten daher, nach dem Auslaufen des Mandats des IRO ihre Zugehörigkeit zu den Volksdeutschen zu beweisen (Volf 1995, S. 427). Die Einbürgerung für NichtDeutschsprachige blieb aber an eine individuelle Überprüfung durch die Behörde gebunden. Das Gesetz sah nicht nur sehr restriktive Aufenthaltsbestimmungen vor, die Einbürgerung war auch sehr teuer. Bis zum 31.3.1961 wurden 300.000 Ausländer eingebürgert, 250.000 davon waren Volksdeutsche (Volf 1995, S. 417). ${ }^{4}$

Der freie Zugang zum Arbeitsmarkt und die einfache Vergabe der Staatsbürgerschaft waren damit die zentralen Integrationsinstrumente für die Volksdeutschen. Da ein großer Teil der Volksdeutschen am Schwarzmarkt beschäftigt war, kann diese Politik durchaus als eine der größten Legalisierungsaktionen der frühen Nachkriegszeit in Europa verstanden werden.

Die Frühphase der Zweiten Republik war somit deutlich von einer Politik der selektiven Integration geprägt, bei der einer forcierten Gleichstellung von Flüchtlingen mit deutscher Muttersprache eine Integrationsverhinderung fremdsprachiger, insbesondere auch jüdischer Flüchtlinge und DPs gegenüberstand. Letztere wurden nicht nur konsequent vom Arbeitsmarkt ausgeschlossen, sondern hatten auch einen viel schwereren Zugang zur Einbürgerung. Während die deutsche Muttersprache von

\footnotetext{
4) Volf nennt als Quelle den Bericht des Bundesministeriums für Inneres an den Nationalrat über die Flüchtlingssituation in den Jahren 1945-1961 und über die Auflösung der Altflüchtlingslager in Österreich, III-46 der Beilagen zu den stenografischen Protokollen des Nationalrats, X.GP, 29.5.1964. FAssmAnN \& MünZ (1995, S. 34) nennen ohne Quellenangabe eine Zahl von 530.000 in Österreich Verbliebenen.
} 
den Alliierten als Zeichen kollektiver Zugehörigkeit zum ehemaligen Kriegsfeind instrumentalisiert wurde, wurden den Volksdeutschen in Österreich kollektiv positive Attribute wie ,,arbeitsam“, „fleißig“ und „loyal“ zugeschrieben. Dagegen galten die fremdsprachigen Flüchtlinge - denen der Arbeitsmarktzugang rechtlich weitgehend verschlossen blieb - als ,,arbeitsscheu“. Die Kenntnis der deutschen Sprache verlor in den 1960er Jahren ihre Bedeutung als Symbol der Zugehörigkeit. Sie wurde erst in den letzten Jahren durch die Integrationsvereinbarung wieder zu einem solchen erhoben.

\section{Arbeitsmarktpolitik in der frühen Nachkriegszeit: der Kampf um den staatlich regulierten Arbeitsmarkt}

Eine der zentralen politischen Konfliktlinien der frühen Nachkriegszeit war die Frage nach der politischen Kontrolle des Arbeitsmarktes. Dabei stießen zwei konträre Konzepte aufeinander. Die Arbeitgebervertreter sowie die ÖVP setzten auf die Selbstregelungskräfte des Marktes. Die Gewerkschaften und die mit ihnen verbündete Sozialdemokratie verfolgte die Idee eines korporatistisch regulierten Arbeitsmarktes, um eine Massenarbeitslosigkeit wie in den 1930er Jahren durch makropolitische Steuerung zu verhindern.

Diese Ideen knüpften einerseits an die Erfahrungen der Ersten Republik an, waren aber andererseits auch Ausdruck einer generellen Polarisierung zwischen marktwirtschaftlichen und staatswirtschaftlichen Positionen der damals noch existierenden großen politischen Lager. Die Entwicklung der Migrationspolitik der 1950er und 1960er Jahre ist in diese Auseinandersetzung eingebettet.

Die wirtschaftspolitische Stabilisierung der ersten Nachkriegsjahre wurde vor allem durch die fünf Lohn-Preis-Abkommen zwischen 1947 und 1951 erreicht. Indem sie die Entwicklung einer Lohn-Preis-Spirale verhinderten und den Unternehmen die Schaffung von Eigenkapital ermöglichten, legten sie die Grundlage für eine langfristig stabile Wirtschaftsentwicklung. Den Arbeitnehmern verlangten die Abkommen Lohnzurückhaltung trotz massiven Wirtschaftswachstums ab, was mehrfach zu Streiks und Widerstand führte (Ludwig et al. 1995). Der Gewerkschaftsbund verlangte als Preis für seine Zustimmung zu den Abkommen die Etablierung einer makroökonomisch orientierten, von ihm mitbestimmten nationalen Arbeitsmarktpolitik, während die Bundeswirtschaftskammer einem marktliberalen Modell anhing. Als einen Teil der Arbeitsmarktsteuerung forderte der Österreichische Gewerkschaftsbund (ÖGB) auch eine Regulierung der Ausländerbeschäftigung durch Ausschüsse bei den Arbeitsämtern, die von den Interessensvertretungen paritätisch beschickt sein sollten. Damit griff der ÖGB auf das bereits in der Ersten Republik etablierte Modell der paritätischen Gestaltung der Ausländerbeschäftigung zurück.

Angesichts der geringen Zuwanderung und des Wirtschaftswachstums ging es bei der Durchsetzung dieser Forderung nicht so sehr um Beschäftigungspolitik als um eine möglichst weitgehende Ausdehnung der eigenen Einflusssphäre in der Arbeitsmarktpolitik. Im Vordergrund stand dabei die Beschäftigung der Österreicher nach 
dem Vorbild des „Inländerarbeitnehmerschutzes“ der Ersten Republik. Dieses Ziel sollte mit der paritätischen Besetzung der Arbeitsämter durch Arbeitgeber- und Arbeitnehmervertreter (seit 1946), der Gründung von Vermittlungsausschüssen für die Ausländerbeschäftigung und der Abschaffung der zuvor nach zehn Jahren erlaubten Bewegungsfreiheit am Arbeitsmarkt für Ausländer erreicht werden. Zudem wurde damit die Ausländerbeschäftigung der Kontrolle des Gesetzgebers entzogen und zur alleinigen Materie der Sozialpartner. Ein wesentlicher Teil der Arbeitsmarktpolitik wurde somit außerhalb der formalen demokratischen Entscheidungsstrukturen entwickelt.

Das Betriebsratsgesetz vom 28.3.1947 schrieb den Status von Ausländern als Arbeitnehmer zweiter Klasse weiter fest. Anders als im Betriebsratsgesetz von 1919, für das die Staatsbürgerschaft kein relevantes Kriterium war, knüpfte das Betriebsratsgesetz von 1947 (BGBl. 97, 2.8.1947) am ständestaatlichen „Werksgemeinschaftsgesetz“ von 1934 an und schloss Ausländer vom aktiven und passiven Betriebsratswahlrecht aus (SEnsenig 1998, S. 372). Damit waren die Eckpfeiler für ein korporatistisches Modell der Arbeitsmarktsteuerung eingeschlagen, in dem Arbeitsmarktpolitik vor allem die Beschäftigung der eigenen Staatsbürger sichern sollte und ausländische Arbeitskräfte als Arbeitnehmer zweiter Klasse angesehen wurden. Es waren damit auch die Weichen für eine dominante Rolle der Sozialpartner in der Migrationspolitik gestellt, die bis in die 1980er Jahre währen sollte.

\section{Gastarbeiteranwerbung und Sozialpartnerschaft}

Zu Mitte der 1950er Jahre kam es im ganzen westlichen Europa zu einer steigenden Nachfrage nach Arbeitskräften. Während die ehemaligen großen Kolonialmächte Frankreich und das Vereinigte Königreich (daneben auch Belgien und die Niederlande) diese vor allem durch Einwanderer aus den Kolonien abdeckten, rekrutierten Deutschland, die Schweiz oder Schweden vor allem in den Nachbarländern und im südeuropäischen Raum. Diese Anwerbung erfolgte nicht einseitig, sondern in Kooperation mit den Herkunftsländern. Diese verfügten wegen massiver Mechanisierung der Landwirtschaft über einen Arbeitskräfteüberschuss und erhofften sich von der Arbeitsmigration eine Dämpfung der Arbeitslosigkeit sowie Deviseneinnahmen durch Rücküberweisungen (ICDUYGU 2006, S. 3).

Verglichen mit Deutschland und der Schweiz erfolgte der Wirtschaftsaufschwung in Österreich um einige Jahre verzögert. In den strukturschwachen südlichen und südöstlichen Bundesländern - Kärnten, Steiermark, Burgenland - blieb die Arbeitslosigkeit hoch. Die Binnenmigranten aus diesen Bundesländern und die Volksdeutschen, denen 1952 der freie Arbeitsmarktzugang eröffnet worden war, bildeten bis in die 1960er Jahre noch ein großes Arbeitskräftereservoir.

Als Ende der 1950er Jahre aber doch ein Mangel an Arbeitskräften spürbar wurde, versuchte die Wirtschaftskammer zuerst, durch Verhandlungen mit deutschen und schweizerischen Firmen die Anwerbung von Österreichern zu stoppen. Als dies nicht gelang, wurde die Anwerbung im Ausland - im europäischen Vergleich relativ spät zu einem zentralen wirtschaftspolitischen Thema (Münz, Zuser \& KYTIR 2003, S. 21). 
Als die Bundeswirtschaftskammer Ende der 1950er Jahre eine Ausweitung der Ausländerbeschäftigung forderte, geriet die seit dem Lohn-Preis-Abkommen etablierte Kooperation der Interessensvertretungen in eine Krise. Der ÖGB und das Sozialministerium beantworteten diese Vorstöße mit der Forderung nach einer aktiven Arbeitsmarktpolitik zur Mobilisierung der inländischen Arbeitskraftreserve (SENSENIG 1998, S. 412).

Im Dezember 1959 hob der Verfassungsgerichtshof (VfGH) die bestehenden ausländerrechtlichen Bestimmungen auf, da er eine Regelung der Ausländerbeschäftigung außerhalb des Parlaments als verfassungswidrig ansah, und forderte den Gesetzgeber auf, die Materie neu zu regeln. Da die reichsdeutsche Ausländerverordnung vom VfGH voll in Kraft gesetzt worden war, bekamen ausländische Arbeitnehmer wieder das Recht, sich nach bestimmter Zeit am Arbeitsmarkt frei zu bewegen, und es wurden ihnen wieder Berufungsrechte gegen negative Bescheide eingeräumt.

Der von Sozialministerium und Gewerkschaft gemeinsam erarbeitete Gesetzesentwurf von 1960 war dagegen von der Idee einer weitgehenden Verhinderung der Ausländerbeschäftigung und einem Verständnis des Arbeitsmarkts als für Staatsbürger reserviertes öffentliches Gut geprägt. Ausländische Arbeitskräfte sollten nur einen im spezifischen Bedarfsfall rekrutierten Puffer am Arbeitsmarkt bilden und keine weiteren Rechte bekommen. Bei Erteilung einer Beschäftigungsbewilligung sollte den Arbeitgebern die Pflicht auferlegt werden, auch eine inländische Arbeitskraft anzulernen oder einen inländischen Lehrling aufzunehmen, ,,sodass die eigene Arbeitskraft keinen Schaden erleide“ (Wollner 1996, S. 50).

Die Bundeswirtschaftskammer schlug stattdessen einen möglichst einfachen Zugang ausländischer Arbeitskräfte vor. ${ }^{5)}$ Dem hielt der ÖGB ein an der Schweiz orientiertes Rotationsmodell entgegen, das gesetzlich regeln sollte, ,,dass die ausländischen Hilfskräfte nach Ende der Saison auch wirklich das Land verlassen“ (zitiert nach WoLLNER 1996, S. 34). Beide Modelle widersprachen der geltenden rechtlichen Grundlage der Ausländerbeschäftigung, die auf einem komplexen Einzelfallverfahren beruhte.

Die Verhandlungen um den Gesetzesentwurf scheiterten. Gleichzeitig wurde der Ruf nach einer flexibleren Regelung der Ausländerbeschäftigung immer lauter. Im September 1961 kam es zum Kompromiss zwischen dem damaligen Gewerkschaftspräsidenten Franz OLAH und dem damaligen Präsidenten der Bundeswirtschaftskammer Julius RAAB. Sie trafen eine Vereinbarung, im nächsten Jahr 47.000 ausländische Arbeitskräfte im Kontingent und ohne Einzelverfahren zuzulassen. Der ÖGB erreichte für seine Zustimmung zur Kontingentierung die Zusage eines vertieften Einflusses im sozialpartnerschaftlichen Unterausschuss für Lohnfragen der Paritätischen Kommission (WIMMER 1985). Ebenso setzte er durch, dass ausländische Arbeitnehmer zwar bei den Lohn- und Arbeitsbedingungen gleichbehandelt werden sollten, jedoch für maximal ein Jahr zugelassen und vor Inländern gekündigt werden mussten. Eine Aufenthaltsverfestigung sollte jedenfalls vermieden werden. „Es ist Ihnen sicher bekannt, dass der ÖGB nach dem Schweizer Muster darauf dringen muss, dass ausländische Arbeitskräfte nach Ablauf der Beschäftigungsgenehmigung Österreich wieder verlassen“, schrieb

\footnotetext{
5) Julius RAAB: Die Kammerorganisation der Gewerblichen Wirtschaft. Ansprache am Kammertag der Bundeskammer anlässlich der Wahl des Präsidenten,26. Mai 1961._http://wko.at/mk/60jahre/Raab_20_05_1961.pdf
} 
Olah an RaAB (Wollner 1996, S. 61f.). So wie in Österreich wurde dieses Rotationsmodell damals in den meisten europäischen Staaten positiv gesehen (ZOLBERG 1991).

Das innenpolitisch bedeutendste Ergebnis des Abkommens war jedoch die Etablierung der Sozialpartnerschaft als spezifisch österreichische Form korporatistischer Politik und ihrer Dominanz in der Arbeits- und Sozialpolitik (TÁlos 2006, S. 434). Die Bundeswirtschaftskammer erkannte nun die Paritätische Kommission für Lohnund Preisfragen, die sie immer abgelehnt und nur als temporäre Notlösung bezeichnet hatte, als Dauereinrichtung an. Damit war die Sozialpartnerschaft fest im politischadministrativen System Österreichs verankert.

\section{Die ungewollte Zuwanderung}

Anders als in Deutschland oder in der Schweiz war die Schwerindustrie in Österreich zu einem großen Teil verstaatlicht. Die „Verstaatlichte Industrie“ unterlag massiver politischer Kontrolle, die bis hin zur Rekrutierung von Mitarbeitern und deren Karriereentwicklung reichte. Wegen des dort großen Einflusses der Gewerkschaft gab es in der „Verstaatlichten“ so gut wie keine ausländischen Beschäftigten. Die Beschäftigung von Ausländern konzentrierte sich dagegen auf das Bauwesen, auf private gewerbliche und industrielle Unternehmen und den Dienstleistungsbereich. Diese Branchen boten aber zumeist weder gute Entwicklungsperspektiven, noch hohe Entlohnung. Für ausländische Arbeitnehmer war Österreich daher anfänglich nicht besonders interessant.

Um genügend Arbeitskräfte nach Österreich holen zu können, schloss die Republik daher Anwerbeabkommen mit Jugoslawien und der Türkei. In Zusammenarbeit mit den Arbeitsmarktbehörden des Entsendelandes gründete die Bundeswirtschaftskammer Anwerbestellen in den großen Städten der beiden Länder. Vor allem in der Türkei kooperierten diese eng mit den deutschen Anwerbestellen, was aber den Effekt hatte, dass Österreich jene Arbeitsmigranten erhielt, die den deutschen Ansprüchen nicht genügten. Noch mehr als die jugoslawische konzentrierte sich daher die türkische Zuwanderung nach Österreich auf ländliche Bevölkerung ohne Arbeitserfahrung in der Industrie. Besser qualifizierte Migranten mit Industriearbeitserfahrung gingen nach Deutschland und in die Schweiz, wo sie ein wesentlich besseres Einkommen erwartete.

Anders als in Jugoslawien mit seinem flächendeckenden Schulsystem war das Schulwesen in den ländlichen Gebieten der Türkei zu dieser Zeit unterentwickelt. Viele türkische Zuwanderer hatten daher keine oder nur rudimentäre Schulbildung (CICEKLI 1998). Da das Rotationsprinzip auch keinen Anreiz zu Bildung und Integration bot, gaben sie ihren niedrigen Bildungsstand zumeist auch an ihre Kinder weiter.

Bis Ende der 1960er Jahre hatte sich aus der Anwerbung ein selbsttragendes Migrationssystem entwickelt. Die Unternehmer wandten sich an ihre ausländischen Mitarbeiter, und diese holten Verwandte und Bekannte nach. Bald stellte sich aber das Rotationsprinzip als unpraktikabel heraus: die Unternehmer waren nicht bereit, die eben erst angelernten Arbeitskräfte jedes Jahr zu ersetzen; und die Sparziele der 
Migranten ließen sich in kurzer Zeit zumeist nicht verwirklichen. Angesichts des Arbeitskräftemangels verlängerten daher die Arbeitsämter die bestehenden Bewilligungen. Die Rückkehr wurde Jahr für Jahr aufgeschoben. Und die Illusion, dass man doch noch einmal zurückkehren werde, wurde auch von der österreichischen Politik verstärkt, die nicht müde wurde, den zeitweiligen Charakter der Arbeitsmigration zu betonen (BAuböck \& Perchinig 2006b, S. 730).

Die im westeuropäischen Vergleich verhältnismäßig schlechte Rechtsstellung der „Gastarbeiter“ am Arbeitsmarkt ermöglichte es, sie in Krisenzeiten schnell abzubauen. In der Krise 1974-1976 wurde etwa 55.000 ausländischen Beschäftigten die Arbeits- und damit die Aufenthaltsgenehmigung entzogen, 1982-1984 weiteren 33.000 (BAUвÖсK \& Perchinig 2006b, S. 730).

Der 1973 beschlossene Anwerbestopp führte jedoch nicht zu einem Rückgang der Einwanderung, sondern zum Anwachsen des Familiennachzugs. Hatten Migranten zuvor damit rechnen können, mehrere Phasen temporärer Arbeitsmigration mit längeren Rückkehrphasen zu kombinieren, mussten sie sich nun zwischen der Rückkehr und der Niederlassung in Österreich entscheiden. Wer die Familie jetzt nicht nachholte, konnte angesichts der Verschärfungen nicht sicher sein, ob ihm das später noch gelingen werde.

In den 1970er Jahren kam es auch erstmals zu einer breiteren öffentlichen Diskussion über die Zuwanderung. Während in Deutschland die Gewerkschaften das Thema „Integration“"vorantrieben und sich für eine bessere rechtliche Stellung der „Gastarbeiter“ einsetzten, sahen die österreichischen Gewerkschaften und die Regierungskoalition aus SPÖ und Freiheitlicher Partei Österreichs (FPÖ) ausländische Arbeitnehmer weiter als Verschubmasse am Arbeitsmarkt an und fokussierten ihre Politik auf die Interessen der - wahlberechtigten - österreichischen Staatsbürger. In einem Artikel in der „Kronen-Zeitung“ vom 6. Februar 1974 stellte etwa der sozialdemokratische Sozialminister Rudolf HäUSER fest, dass die „Österreicher“ durch die Beschäftigung von Gastarbeitern niemals einen Nachteil erleiden würden (BRATic 2002, S. 6). Der öffentliche Diskurs kippte in der Folge, die „Gastarbeiter“ wurden zunehmend als Belastung dargestellt.

Angesichts der Krise einigten sich die Sozialpartner auf Druck der Gewerkschaft im Jahr 1974 auf ein Einfrieren der Ausländerbeschäftigung auf den Stand vom Oktober 1973. Neben der Reduzierung der Kontingente sollten vor allem schärfere Kontrollen einen Rückgang bewirken. Die Verwaltung wurde von den Sozialpartnern aufgefordert, „strenger den Wohnungsnachweis zu prüfen und die fremdenpolizeilichen Maßnahmen einzusetzen, um die Mißstände in der Quartierung und der Touristenbeschäftigung zu beseitigen“" (MATUSCHEK 1986, S. 182).

Doch auch österreichische Gastarbeiter waren vom Anwerbestopp betroffen. Von den rund 101.000 österreichischen Gastarbeitern, die 1973 in Deutschland arbeiteten, mussten bis zum Jahr 1977 26.100 Deutschland verlassen. Allein im Jahr 1975 kehrten rund 15.000 Gastarbeiter nach Österreich zurück und suchten in Österreich Arbeit, was als zusätzliches Argument für die Reduktion der Ausländerbeschäftigung im Land galt (SEnsenig 1998, S. 421). 
Das 1975 verabschiedete Ausländerbeschäftigungsgesetz verschärfte den Inländerprimat, und den ausländischen Beschäftigten wurde das ihnen bisher zustehende Recht, gegen die Versagung und den Widerruf der Arbeitserlaubnis Rechtsmittel zu ergreifen, genommen. Als Verbesserung wurde das Institut des Befreiungsscheins eingeführt, das Ausländern nach acht Jahren Beschäftigung eine auf zwei Jahre befristete Freizügigkeit am Arbeitsmarkt gewährte. Insgesamt war das Gesetz jedoch von der Intention geprägt, Ausländer als Arbeitnehmer zweiter Klasse zu behandeln und ihre Niederlassung und Integration so weit wie möglich zu verhindern.

Besonders deutlich wurde dies beim 1974 beschlossenen Arbeitsverfassungsgesetz, das zwar Ausländern das aktive, nicht aber das passive Betriebsratswahlrecht zugestand. Es verhinderte somit, dass Interessen der Arbeitsmigranten wirksam gewerkschaftlich vertreten wurden (BAuBöck \& Perchinig 2006b, S. 731). Dieses Gesetz hatte auch die Unterstützung der Gewerkschaften. Es stand in krassem Gegensatz zu Deutschland, wo im Jahr 1973 die Staatsbürgerschaft als Kriterium für die Wahl zum Betriebsrat abgeschafft wurde (Hunn 2004) und in der Folge viele „Gastarbeiter“ Karriere in den Gewerkschaften machten. In Österreich blieb dagegen Migranten der wichtigste Rekrutierungskanal für Funktionen in der Gewerkschaft und in den Arbeiterkammern verschlossen. Es finden sich daher in Österreich in den Leitungspositionen des ÖGB und der Arbeiterkammer bis heute kaum Einwanderer.

Strukturell verfestigte das Ausländerbeschäftigungsgesetz die Sozialpartnerdominanz. Es band die Präsidentenkonferenz der Landwirtschaftskammern in das System ein und billigte dem ÖGB einen zweiten Vertreter im Ausländerausschuss zu. Dieser Ausschuss wurde gesetzlich verankert und erhielt die Kompetenz zur Festlegung von Höchst- und Landesverhältniszahlen von Ausländern. Damit waren die korporatistische Steuerung des Arbeitsmarktes und die Einbindung der Ausländerbeschäftigung in diese wieder einen Schritt voran gekommen. „Durch die Ankopplung des Ausländerausschusses an die zentrale Koordinierungsstelle der Arbeitsmarktpolitik, den Beirat und das Sozialministerium (wurde) (...) die Ausländerpolitik an den jährlich festgelegten arbeitsmarktpolitischen Schwerpunktprogrammen ausgerichtet" 1985 Helga Matuscheк, eine der ersten österreichischen Migrationsforscherinnen, diese Entwicklung (Matuscher 1986, S. 189).

Als Ausgleich zu den Verschärfungen bei der Ausländerbeschäftigung sollte nach dem Wunsch der Regierung zumindest die Einbürgerung etwas erleichtert werden. Dazu waren eine Kürzung der zehnjährigen Wartefrist, die Anerkennung einer doppelten Staatsbürgerschaft und Erleichterungen beim Einkommensnachweis geplant. Die erforderlichen „besonderen Verdienste für die Republik“ sollten durch „Verdienste für die Republik“ ersetzt werden. Gegen diese Vorschläge leistete die ÖVP heftigen Widerstand und warf der SPÖ vor, um Wähler zu buhlen. Da zur Umsetzung dieser Reform eine Zwei-Drittel-Mehrheit nötig gewesen wäre, kam sie nicht zustande. Es kam nur zu einigen minimalen Erleichterungen bei der Einbürgerung von in Österreich geborenen Kindern ausländischer Eltern und zu Verbesserungen bei der Wiedererlangung der österreichischen Staatsbürgerschaft für die Opfer des Holocausts (ÇINAR \& WALRAUCH 2006). 


\section{Von der Sozialpartnerschaft ins Parlament}

Wie im Jahr 1959 wurde auch zu Mitte der 1980er Jahre der Verfassungsgerichtshof (VfGH) zu einem entscheidenden Akteur bei der Durchsetzung eines langfristig wirksamen Politikwandels. Aufgrund einer Beschwerde hob er im Jahr 1985 das seit 1954 geltende Fremdenpolizeigesetz wegen mangelnder Konformität mit den Bestimmungen der Europäischen Menschenrechtskonvention zum Schutz des Privat- und Familienlebens auf. Er bereitete damit den Weg für einen Perspektivenwechsel weg von der alleinigen Arbeitsmarkt- hin zu einer Niederlassungsperspektive, da nun das Recht auf Familienleben gegen die arbeitsmarktpolitischen Gestaltungsinteressen abzuwägen war (B AubÖск \& PerChINIg 2006b, S. 731). Der Entscheid des Verfassungsgerichtshofs bewirkte auch eine Änderung des institutionellen Rahmens. Neben dem Sozialministerium wurde nun auch das Innenministerium, das zuvor nur in der Flüchtlingspolitik aktiv war, zu einem Akteur in der Arena der Ausländerpolitik, die aber weiter von den Sozialpartnern dominiert blieb.

Auch im Staatsbürgerschaftsgesetz erfolgten aufgrund der nötigen Anpassung an die Internationale Konvention gegen Frauendiskriminierung Änderungen, insbesondere bei der Übertragung der Staatsbürgerschaft auch in der mütterlichen Linie. Das Abstammungsprinzip wurde jedoch beibehalten. Entgegen dem europäischen Trend wurde auch eine doppelte Staatsbürgerschaft nicht zugelassen. Durch die Gleichstellung der Geschlechter verschwand allerdings auch ein bisheriges Privileg der Frauen. Hatten sie bis dahin allein durch die Eheschließung und ohne weitere Bedingungen die österreichische Staatsbürgerschaft erhalten, so unterlagen nun auch Ehefrauen von Österreichern den allgemeinen Einbürgerungsbedingungen (ÇINAR \& W ALRAUCH 2006, S. 28).

Das österreichische Parteiensystem erlebte in den 1980er Jahren massive Umbrüche, die langfristig auch bedeutsam für die Weiterentwicklung der Migrationspolitik wurden. Im Jahr 1986 übernahm Jörg HAIDER die Führung der FPÖ und leitete den Wandel einer ehemaligen Honoratiorenpartei mit einem Stimmenanteil um die 5\% zu einer rechtspopulistischen Massenpartei mit starker Verankerung bei Arbeitern und Wahlergebnissen bis zu 30\% ein. Die 1980er Jahre waren aber auch das Geburtsjahrzehnt der Grünen, die sich als vierte Partei im politischen Spektrum etablierten und ins Parlament einzogen. Beide Parteien waren nicht in das Elitenkartell der Sozialpartnerschaft eingebunden und benötigten zur Profilierung Themen, die noch nicht von den damaligen Großparteien besetzt waren. Die Migrationspolitik, die bis dahin von den Sozialpartnern fast unter Ausschluss der Öffentlichkeit gestaltet und durch die Verschränkung der Gewerkschafts- und Wirtschaftsfunktionäre mit SPÖ bzw. ÖVP im Parlament praktisch „durchgewunken“ worden war, bot sich dazu an.

Zuvor fast nur unter einer Arbeitsmarktperspektive diskutiert, wurde das Thema „Migration“"von den Grünen zunehmend unter menschenrechtlichen Gesichtspunkten aufgegriffen, während die FPÖ das Thema für nationalistische Mobilisierung nutzte (Bauböck \& Perchinig 2006b, S. 732; Kraler \& Stepien 2006, S. 4). 
Paradoxerweise ermöglichte es gerade der Versuch der Grünen und der FPÖ, die Thematik ins Parlament zu verlagern, den damaligen Großparteien SPÖ und ÖVP weiter an der etablierten Kooperation im Rahmen der Sozialpartnerschaft festzuhalten. Sie konnten sich nämlich gegenüber den aktionistisch agierenden Kleinparteien als sachorientierte und erfahrene Akteure zeigen und die Fortsetzung der bisherigen Politik als Weg der Mitte darstellen.

Anfang der 1990er Jahre wurden vermehrt Konzepte einer „kompensatorischen Einwanderungspolitik“ in die Diskussion eingebracht. Sie liefen darauf hinaus, die Zuwanderung wie die großen Einwanderungsländer mittels Kontingentierung zu regeln und den Zuwanderern stärkere Rechtssicherheit zu bieten. Diese von der Regierung VRANITZKY unterstützten Konzepte scheiterten jedoch am Widerstand der Gewerkschaften, die den Bereich der Arbeitsmigration nicht aus ihrer Kontrolle entlassen wollten.

So brachte die 1988 beschlossene Novellierung des Ausländerbeschäftigungsgesetzes zwar eine Verbesserung der Freizügigkeit. Gleichzeitig verschärfte die Novelle aber durch das Festschreiben einer Bundeshöchstzahl (Quotierung des Gesamtanteils der Ausländer am heimischen Arbeitsmarkt) die Rolle der Arbeitsmigranten als Reservearmee des Arbeitsmarktes drastisch. Effekt dieser Regelung war eine Zugangssperre zum Arbeitsmarkt für zahlreiche legal in Österreich lebende Ausländer (vor allem ausländische Frauen nach der Karenz) und damit die Verarmung ausländischer Haushalte (GÄCHTER 1995). Trotz der Verfestigung der Einwanderung wurde somit die im Wesentlichen gescheiterte Gastarbeiterpolitik weiter festgeschrieben.

\section{Migrationspolitik nach dem Ende des Kalten Krieges}

Die politischen Umbrüche in Europa sowie die steigende Nachfrage nach Arbeitskräften führten zwischen 1987 und 1994 zu einem massiven Anwachsen der Wanderungsströme. Die Zahl der in Österreich lebenden Ausländer verdoppelte sich in diesem Zeitraum von 326.000 auf 713.000. Mit einer Nettozuwanderungsrate von $1 \%$ pro Kopf der Bevölkerung lag Österreich im europäischen Spitzenfeld (BAUBöcK \& Perchinig 2006b, S. 732). Diese Entwicklungen wurden in der Öffentlichkeit vor allem als Bedrohung wahrgenommen.

Nicht zuletzt aufgrund des wachsenden Zustroms zur FPÖ, die sich klar gegen Einwanderung positionierte, wuchs in der Regierung die Überzeugung, dass eine Neuregelung des Migrationsrechts nötig wäre. Unter Federführung des zuständigen Sektionschefs im Innenministerium, Manfred MATZKA, wurden Anfang der 1990er Jahre sowohl das Asylrecht neu geregelt wie ein neues Fremden- und Aufenthaltsrecht geschaffen. Das Innenministerium war nun die entscheidende Instanz für die Gestaltung der Migrationspolitik geworden. Es ging vom Prinzip der Migrationsregelung durch Arbeitsmarktpolitik ab und implementierte erstmals eine direkte Einwanderungssteuerung, indem die Höchstzahl der zu vergebenden Aufenthaltstitel festgesetzt wurde. 
Der Zugang zum Aufenthaltsrecht wie zum Asyl wurde in den neuen Gesetzen deutlich erschwert. Hatte schon bisher das „Ausländerbeschäftigungsgesetz“ einen Höchstanteil von erlaubten ausländischen Beschäftigten festgelegt, so wurde im 1993 in Kraft getretenen Fremdenrechtspaket zusätzlich eine Höchstzahl von jährlich zu vergebenden Aufenthaltsgenehmigungen eingeführt. Die Aufenthaltsgenehmigung war jedoch nicht mit einem Recht auf Beschäftigung verbunden. Anträge auf Einwanderung mussten grundsätzlich aus dem Ausland gestellt werden. Erst nach mehreren befristeten Genehmigungen konnte nach frühestens fünf Jahren eine unbefristete Bewilligung erteilt werden. Fristversäumnisse führten zum Verlust des Aufenthaltsrechts, was vor allem viele bereits langansässige Migranten traf, die einen Termin übersehen hatten und nun unter sehr restriktiven Bedingungen einen Antrag auf Neuzuwanderung aus dem Ausland stellen mussten.

Zusätzlich erschwerten detaillierte Bestimmungen über den Nachweis einer ortsüblichen Wohnung den Familiennachzug, der durch Einrechnung in die Quote massiv behindert wurde. Da bis zu einer Novelle im Jahr 1995 auch die im Inland geborenen Kinder in die Höchstzahl der Aufenthaltsgenehmigungen eingerechnet wurden, wurden nun sogar Neugeborenen Ausweisungsbescheide wegen illegalen Aufenthalts zugestellt (KöNig \& STADLER 2003).

Ein Jahr später wurde die „Bundeshöchstzahl“ des Ausländerbeschäftigungsgesetzes auf $8 \%$ des Arbeitskräfteangebots gesenkt und damit der Zugang zum Arbeitsmarkt weiter erschwert.

Im Zusammenspiel mit dem Ausländerbeschäftigungsgesetz bildete das Fremdenrecht nun ein System stufenweiser Ausschließung und Destabilisierung des Aufenthaltsstatus, das darauf hinzielte, ausländische Arbeitnehmer nicht nur, wie schon zuvor, bei Bedarf vom Arbeitsmarkt entfernen zu können, sondern ihnen auch bei lang andauernder Arbeitslosigkeit die Grundlage für den Weiteraufenthalt in Österreich zu entziehen und den Familiennachzug möglichst einzuschränken (KöNIG \& STAdLER 2003).

Die FPÖ nützte die massive Neuzuwanderung, um 1993 unter dem Motto „Österreich zuerst" ein Volksbegehren für eine restriktivere Zuwanderungs- und Asylpolitik aufzulegen. Dieser nur von 7,4\% der Wahlberechtigten unterstützten Kampagne stellte sich eine breite Koalition gegen Rassismus und Ausländerfeindlichkeit entgegen, die 1993 die größte Kundgebung der Zweiten Republik, das „Lichtermeer“, organisierte. Bei den Nationalratswahlen 1994 legte die FPÖ aber um 6\% zu und entwickelte in der Folge einen scharf ausländerfeindlichen und gegen den anstehenden EU-Beitritt Österreichs gerichteten Oppositionskurs. Innerhalb der größeren Regierungspartei SPÖ wuchs der Unmut über die restriktive Migrationspolitik. Insbesondere in Wien, wo 1992 mit dem „Wiener Integrationsfonds“ die erste städtische Integrationseinrichtung Österreichs von einer SPÖ-Stadtregierung ins Leben gerufen worden war, forderten einflussreiche Teile der SPÖ einen milderen Kurs (KOLLER 1998).

Nach den Wahlen des Jahres 1995 zog mit dem Liberalen Forum eine weitere (neben den Grünen) in Migrationsfragen liberale Partei ins Parlament ein. In der neuen Regierung wurde der dem rechten Parteiflügel zugehörige und als Vertreter einer "Law-and-Order"-Politik geltende SPÖ-Innenminister Franz LöschNAK von Caspar 
EINEM abgelöst, der noch als Mitarbeiter der Arbeiterkammer Wien im Jahr 1990 SPÖ-intern mit „Alternativen Optionen der Zuwanderungspolitik“ den Schwenk zu einer Einwanderungspolitik mit möglichst schneller rechtlicher Gleichstellung der Migranten skizziert hatte (EINEM 1990).

Nachdem die österreichischen Höchstgerichte wesentliche Bestimmungen des Fremden- und Ausländerbeschäftigungsgesetzes aufgehoben hatten und Österreich auch mehrfach vom Europäischen Gerichtshof für Menschenrechte wegen seiner Fremdengesetze verurteilt worden war, verbesserte die unter Minister EINEM entstandene Fremdenrechtsnovelle 1997 die Rechtslage langansässiger Migranten deutlich. Sie führte ein sicheres Aufenthaltsrecht nach achtjährigem Aufenthalt ein und schützte insbesondere Jugendliche der „Zweiten Generation“ vor Abschiebungen.

Die Verurteilung Österreichs durch den Europäischen Gerichtshof für Menschenrechte im Fall „GAYGuSUZ gegen Österreich“ (EuropäISCHER GERICHTSHOF FÜR MENSCHENRECHTE 1996, S. 135) erzwang die weitgehende Aufhebung der Diskriminierung von Nicht-EU-Ausländern beim Zugang zu den Leistungen der Arbeitslosenversicherung (Notstandshilfe) und führte damit zu einem ersten Bruch des Inländerprimats in der Sozialpolitik. Das Sozialressort konnte mit Unterstützung der Arbeitnehmervertretung jedoch eine von EINEM intendierte Harmonisierung von Aufenthaltsrecht und Arbeitsmarktzugang blockieren und damit den eigenen Einfluss weiter aufrechterhalten.

Auch das Staatsbürgerschaftsrecht wurde in den späten 1990er Jahren grundlegend reformiert. Bis zu seiner Novellierung im Jahr 1998 gab das Gesetz den Behörden weitgehende Ermessensspielräume. Aufgrund der föderalistischen Struktur Österreichs, die sich auch im Staatsbürgerschaftsrecht widerspiegelte, unterschieden sich zudem die Einbürgerungsbedingungen in den Ländern deutlich.

In Wien hatte es in der regierenden SPÖ Anfang der 1990er Jahre eine Debatte um die Einführung eines kommunalen Ausländerwahlrechts gegeben. Dieser Vorschlag fand aber keine Mehrheit. Stattdessen beschloss die Wiener Landesregierung, ihre Spielräume bei der Einbürgerung zu nutzen. Sie senkte die Wartefristen und erleichterte die sonstigen Einbürgerungsbedingungen. Zwischen 1989 und 1994 stieg die Zahl der Personen, die ,,aus besonderen Gründen“ in Wien eingebürgert wurden, von 735 auf 2.028. Im gleichen Zeitraum betrafen zwischen $63 \%$ und $80 \%$ aller erleichterten Einbürgerungen in Österreich das Land Wien (ÇINAR \& WALRAUCH 2006, S. 39f.).

Die liberale Wiener Einbürgerungspolitik stieß bald auf wachsende Kritik vor allem der FPÖ, aber auch der ÖVP, die in dieser Frage bald tonangebend wurde. Im Jahr 1996 stellte die ÖVP einen Entwurf für ein neues Staatsbürgerschaftsrecht vor, der als Einbürgerungsbedingungen eine 15-jährige Aufenthaltsdauer, deutsche Sprachkenntnisse und die Teilnahme an Integrationskursen enthielt. Die FPÖ forderte in diesem Zusammenhang eine Verfassungsbestimmung, dass Österreich kein Einwanderungsland sei, und verlangte eine Beschränkung der jährlichen Einbürgerungen auf 0,5\% der Bevölkerung des jeweiligen Bundeslandes. Auch die Grünen und Liberalen legten entsprechende Entwürfe vor und forderten die Senkung der für die Staatsbürgerschaft notwendigen Aufenthaltsfrist auf fünf Jahre und die Anerkennung der Doppelstaatsbürgerschaft. Die SPÖ entwickelte zwar keinen eigenen Entwurf, doch forderte die Wiener SPÖ die Anerkennung der Doppelstaatsbürgerschaft bis zum Zeitpunkt der 
Volljährigkeit. Die SPÖ-ÖVP-Koalition einigte sich schließlich darauf, die Grundstrukturen des Einbürgerungsrechts unverändert zu lassen, jedoch die Vollziehung in den Bundesländern zu harmonisieren und erstmals deutsche Sprachkenntnisse als Bedingung für die Einbürgerung festzuschreiben, ohne jedoch deren Überprüfung genauer zu regeln (ÇINAR \& W ALRAUCH 2006, S. 40f.).

In den Meinungsumfragen stieg die Popularität der FPÖ Ende der 1990er Jahre deutlich. Nach dem Rücktritt VRANITZKY's als Bundeskanzler wurde unter seinem Nachfolger Viktor KLIMA 1997 der am rechten Rand der SPÖ angesiedelte Karl SCHLÖGL neuer Innenminister. Er hatte sich auch wiederholt für eine Zusammenarbeit mit der FPÖ ausgesprochen. Die kurze liberale Phase der Migrationspolitk war damit beendet.

\section{9 Österreichische Migrations- und Integrationspolitik in der Europäischen Union}

Hatte das Urteil im Fall Gaygusuz der Diskriminierung von Ausländern im Bereich der Arbeitslosenversicherung ein Ende gemacht, so geriet mit dem EU-Beitritt Österreichs im Jahr 1995 der Inländerprimat am Arbeitsmarkt ins Schwanken.

Anders als in Österreich, das ein korporatistisches Modell der Arbeitsbeziehungen etabliert hatte, beruhte die Arbeitsmarktpolitik der EU auf einem marktwirtschaftlichen Zugang. Eine durch Staatszugehörigkeit weitgehend ungestörte Konkurrenz sollte das Geschehen am Arbeitsmarkt prägen, nicht die Trennung in Inländer/Insider und Ausländer/Outsider, die die österreichische Arbeitsmarktpolitik charakterisierte (BIFFL 2001). Wie schon in den Jahren zuvor, waren auch hier wieder die Höchstgerichte entscheidende Akteure bei der Durchsetzung eines Paradigmenwechsels.

Der erste Anlassfall für einen Konflikt, bei dem Österreich nach hinhaltendem Widerstand das EU-Recht akzeptieren musste, war die Umsetzung des Assoziationsrechts, insbesondere des Assoziationsabkommens zwischen der Europäischen Wirtschaftsgemeinschaft (EWG) und der Türkei aus 1963') und der zugehörigen Urteile des Europäischen Gerichtshofs (EuGH) (CICEKLI 2004), wonach türkischen Arbeitnehmern unter bestimmten Voraussetzungen am Arbeitsmarkt ähnliche Rechte wie Unionsbürgern zu gewähren sind. Nachdem das zuständige Sozialministerium die Gültigkeit der assoziationsrechtlichen Bestimmungen lange verneint hatte, setzte der Verwaltungsgerichtshof die Umsetzung des Acquis in einer Reihe von Urteilen durch (Juen, Perchinig \& Volf 1998, S. 211).

Noch breitere Wirkung hatte die Verurteilung des Ausschlusses von türkischen Arbeitnehmern vom passiven Betriebsrats- und Arbeiterkammerwahlrecht als rechtswidrig durch den EuGH im Jahr 2003. 7) Dieses Urteil machte den Weg für eine Klage der Europäischen Kommission gegen Österreich frei, in der diese den Ausschluss von Nicht-Unionsbürgern aus Ländern mit Abkommen mit den Europäischen Gemein-

\footnotetext{
6) 64/732/EWG, 64/733/EWG, Abl. 64/217.

7) Urteil des Gerichtshofes (Sechste Kammer) vom 8. Mai 2003, C-171/01.
} 
schaften vom passiven Arbeiterkammerwahlrecht als Vertragsverletzung wertete. Nachdem Österreich vom EuGH in dieser Angelegenheit verurteilt worden war ${ }^{8)}$ und ein Ausschluss von Migranten aus Ländern ohne entsprechende Abkommen mit den Europäischen Gemeinschaften nicht machbar erschien, wurden Anfang 2006 das Arbeiterkammer- sowie das Arbeitsverfassungsgesetz entsprechend geändert, sodass nun alle Drittstaatsangehörigen das passive Wahlrecht bei Arbeiterkammer- und Betriebsratswahlen besitzen.9)

Diese Entscheidungen markierten den Anfang vom Ende des Paradigmas vom Arbeitsmarkt als öffentlichem Gut nur für eigene Staatsbürger. Zwar setzte Österreich bei der Erweiterung der EU im Jahr 2004 noch lange Übergangsfristen durch, doch mit der Umsetzung des Acquis kam es zu einer weitgehenden rechtlichen Angleichung der Rechtsstellung langansässiger Nicht-EU-Bürger am Arbeitsmarkt. Neben einer - juristisch oft bewundernswert spitzfindigen - Umsetzung der EU-Direktiven auf Minimalniveau versuchte Österreich in der Folge, direkten Einfluss auf Entscheidungen im Rat der EU-Innenminister zu gewinnen. Es wurde mit seinen Positionen zum Vorreiter einer restriktiven Migrationspolitik in Europa (PERCHINIG 2006a).

\section{Die Migrationspolitik der ÖVP/FPÖ-Koalitionsregierung}

Eine spezifische europapolitische Konstellation entstand im Frühjahr 2000, als ÖVP und FPÖ eine Regierungskoalition bildeten. Dies erregte international großes Aufsehen und hatte ein Einfrieren der bilateralen Kontakte der anderen EU-Staaten zu Österreich zur Folge (,Sanktionen“). Wesentlicher Grund dafür war die Einschätzung der EU-Mitgliedsstaaten, dass es sich bei der FPÖ um eine fremdenfeindliche Partei handle, die sich nicht ausreichend vom Nationalsozialismus distanziert hätte.

Anders als international erwartet, kam es aber in der ,schwarz-blauen“ Koalition ${ }^{10)}$ anfangs kaum zu Änderungen der bisherigen Politik. Die Regierung Schüssel I liberalisierte den Arbeitsmarktzugang für Migranten mit zumindest fünfjährigem legalen Aufenthalt und schuf eine Arbeitsgenehmigung aus sozial-humanitären Gründen, die vor allem Migrantinnen nach der Familienphase den Einstieg in den Arbeitsmarkt erleichterte (KöNIG \& STADLER 2003, S. 236). Der zweite Akzent betraf einerseits die Begrenzung der arbeitsbezogenen Einwanderung auf „Schlüsselkräfte“11) und deren Familien, andererseits die Ausweitung der zuvor auf 5.500 jährliche Genehmigungen begrenzten Saisonbeschäftigung auf 15.000 (PERCHINIG 2006b, S. 296).

\footnotetext{
8) Urteil des Gerichtshofes (Zweite Kammer) vom 16.9.2004, C-465/01.

9) BGB1. I Nr. 4/2006.

${ }^{10)}$ In ihrer letzten Phase bildete nicht mehr die FPÖ den Regierungspartner der ÖVP, sondern das von der FPÖ abgespaltete BZÖ (Bündnis Zukunft Österreich). Wenn von der gesamten Regierungsperiode des Kabinetts Schüssel II die Rede ist, wird daher zum umfassenden Ausdruck „schwarz-blaue Koalition“ gegriffen.

11) Definiert als Personen mit einem Einkommen in der Höhe von zumindest $60 \%$ der Höchstbeitragsgrundlage in der Sozialversicherung.
} 
Die öffentliche Diskussion der ersten Jahre der „schwarz-blauen“ Koalition war jedoch nicht von der Arbeitsmigration, sondern vom FPÖ-Vorschlag einer „Integrationsvereinbarung“ dominiert, welche um langfristigen Aufenthalt ansuchende Ausländer zum Besuch eines Sprach- und Integrationskurses verpflichten sollte. Am 13.8.2001 präsentierte der Innenminister den Entwurf eines neuen „Integrationsvertrags“ erstmals im Ministerrat und bezog sich dabei vor allem auf das niederländische Vorbild. Anders als in den Niederlanden, wo ein 600-stündiger Sprachkurs ein gewisses Mindestniveau an Sprachkenntnissen erbringen sollte, sollte die Kursdauer in Österreich aber auf 100 Stunden beschränkt bleiben (Rohsmann 2006). Trotz heftiger Kritik der Oppositionsparteien kam es mit der Reform des Fremdenrechts im Jahr 2002 zur Einführung dieser verpflichtenden Sprachkurse. In der Praxis blieb die Integrationsvereinbarung aber symbolische Politik. Die per 1.1.2003 in Kraft getretene Fremdenrechtsnovelle schrieb zwar die Verpflichtung zum Sprachkursbesuch als Voraussetzung für einen langfristigen Aufenthaltstitel fest, sah allerdings derart viele Ausnahmen vor, dass bis Juni 2004 nur ca. 10\% der rund 118.000 Personen umfassenden Zielgruppe diese Kurse besucht hatten (Die Presse, 17.3.2005, S. 3).

Im öffentlichen Diskurs löste das Thema „Integration“ nun die langjährige Fokussierung auf den Arbeitsmarkt ab. War in den 1990er Jahren unter „Integration“ vor allem rechtliche Gleichstellung - also ein Entgegenkommen der aufnehmenden Gesellschaft - verstanden worden (Rohsmann 2006, S. 75), so verschob sich die Bedeutung des Wortes nun hin zu einer Anpassungsleistung der Migranten. Dies kam auch in der Debatte um die Integrationskurse zum Ausdruck. Diese sollten, so betonte die FPÖ, vor allem auch der „Feststellung der Integrationswilligkeit“ der Migranten dienen (Rohsmann 2006, S. 75).

Dieser Diskurs vermischte sich bald mit einem Diskurs über „gemeinsame Werte“ und der Integrationsfähigkeit des Islams. Insbesondere nach den Terroranschlägen in London und Madrid und nach der Verhaftung von in Österreich aufgewachsenen, für eine islamistische Terrororganisation aktiven jungen Migranten der Zweiten Generation, bekam diese Debatte eine dezidiert sicherheitspolitische Orientierung. Besonders gut sichtbar wurde dieser Wandel in Wahlkämpfen. Waren in den 1990er Jahren noch Drogenhandel und Kriminalität die wesentlichen negativen Stereotypisierungen, so zeigten besonders die Wahlkämpfe zu den Nationalratswahlen 2008 und zum Europäischen Parlament im Jahr 2009 eine Zuspitzung auf das Feindbild Islam (BunzL \& HAFEZ 2009).

Das Kabinett Schüssel II gab sich ein breiteres migrationspolitisches Programm und kündigte Reformen des Asyl- und Staatsbürgerschaftsrechts an. Die Asylpolitik dominierte auch die öffentliche Debatte. Im Bereich des Staatsbürgerschaftsrechts hatte die FPÖ vor allem ab Herbst 2004 Erschwernisse bei der Einbürgerung gefordert. Hintergrund dafür war das massive Anwachsen des Familiennachzugs zu häufig erst vor Kurzem Eingebürgerten türkischer oder auch ex-jugoslawischer Herkunft. Da der Familiennachzug zu Nicht-EU-Bürgern durch Quoten massiv eingeschränkt, zu Österreichern jedoch außerhalb der Quotierung möglich war, hatte die Einbürgerung eine wichtige einwanderungspolitische Dimension erhalten. Allein im Jahr 2004 hatten rund 23.300 Personen ihr Aufenthaltsrecht als Familienangehörige von Österreichern erlangt (Bundesministerium FÜr InNeres 2004). Die Novelle des Staatsbürgerschafts- 
rechts im Jahr 2005 ${ }^{12)}$ sowie die zugehörige Staatsbürgerschaftsprüfungsverordnung ${ }^{13)}$ erhöhten die Hürden bei der Einbürgerung, insbesondere in Bezug auf die Anrechnung des Aufenthalts im Land und die nötigen Einkommenshöhen. Außerdem hob sie die Gebühren für die Einbürgerung so deutlich an, dass die Einbürgerung in Österreich zu einer der teuersten in Europa wurde (ÇINAR \& WaLRAUCh 2006, S. 49 ).

Dem internationalen Trend folgend, verlangt das neue Staatsbürgerschaftsrecht nun auch das Bestehen einer Deutsch-Prüfung sowie einer Prüfung über die „Grundkenntnisse der demokratischen Ordnung der Republik Österreich und (die) Geschichte Österreichs“" (PerChINIG 2009, im Druck). Mit Dänemark, Frankreich, Griechenland, den Niederlanden und dem Vereinigten Königreich bildet Österreich damit einen Block von EU-Staaten, die ihr Staatsbürgerschaftsrecht in den letzten Jahren restriktiver gestalteten (B AUBÖCK \& PerChINIG 2006b).

\section{Die Vergemeinschaftung der europäischen Migrationspolitik und ihre Auswirkungen}

Mit dem Vertrag von Amsterdam (1997/99) war es zu einer Vergemeinschaftung der Integrationspolitik auf EU-Ebene gekommen. Grundsatz dieser Politik war die in den Schlussfolgerungen des Europäischen Rats von Tampere festgehaltene Überlegung, die Rechtsstellung von legal im Land lebenden Nicht-EU-Bürgern so weit wie möglich jener der Staatsbürger anzunähern:

„21. Die Rechtsstellung von Drittstaatsangehörigen sollte der Rechtsstellung der Staatsangehörigen der Mitgliedstaaten angenähert werden. Einer Person, die sich während eines noch zu bestimmenden Zeitraums in einem Mitgliedstaat rechtmäßig aufgehalten hat und einen langfristigen Aufenthaltstitel besitzt, sollte in diesem Mitgliedstaat eine Reihe einheitlicher Rechte gewährt werden, die sich so nahe wie möglich an diejenigen der EU-Bürger anlehnen; z.B. das Recht auf Wohnsitznahme, das Recht auf Bildung und das Recht auf Ausübung einer nichtselbständigen oder selbständigen Arbeit sowie der Grundsatz der Nichtdiskriminierung gegenüber den Bürgern des Wohnsitzstaates. Der Europäische Rat billigt das Ziel, daß Drittstaatsangehörigen, die auf Dauer rechtmäßig ansässig sind, die Möglichkeit geboten wird, die Staatsangehörigkeit des Mitgliedstaats zu erwerben, in dem sie ansässig sind.“ (EuropäISChER RAT VON TAMPERE 1999)

In Umsetzung dieses Auftrags entwickelte die Kommission Richtlinienentwürfe zur Einwanderung aufgrund von Arbeitsaufnahme, ${ }^{14)}$ zur Familienzusammenführung ${ }^{15}$

\footnotetext{
12) BGB1. I Nr. 37/2006, 22.3.2006.

${ }^{13)}$ BGB1. II Nr. 138/2006, 3.4.2006

14) KOM (2001) 386 endg.

15) KOM (1999) 638 endg.
} 
und zur Rechtsstellung von langansässigen Drittstaatsangehörigen. ${ }^{16)}$ Die Entwürfe übertrugen im Wesentlichen die bestehenden Bestimmungen für Unionsbürger auf Drittstaatsangehörige.

Die „Tampere-Prinzipien“ mit ihrem Impetus der weitgehenden Gleichstellung von legal ansässigen Drittstaatsangehörigen und Staatsbürgern standen von Anfang an in einem Spannungsverhältnis zum dominant sicherheitspolitischen Hintergrund der Migrationspolitik im Europäischen Rat und blieben großteils Rhetorik. Die Verschiebung der politischen Mehrheiten im Rat in den späten 1990er Jahren, der Konjunkturrückgang in Europa und die Terroranschläge in New York und Madrid führten zu einer deutlichen Refokussierung des Migrationsdiskurses auf sicherheitspolitische Agenden und zu einer Rücknahme der Gleichstellungsagenda (Bendel 2007; FAist 2006). Der Richtlinienentwurf über die Einreise- und Aufenthaltsbedingungen von Drittstaatsangehörigen zum Zweck der Erwerbstätigkeit ${ }^{17)}$ scheiterte überhaupt. Die Richtlinienentwürfe zur Familienzusammenführung, zum Rechtsstatus von langansässigen Drittstaatsangehörigen und zum Familiennachzug fanden erst nach wesentlichen Änderungen die Zustimmung der Innenminister, wobei sich insbesondere Deutschland, die Niederlande und Österreich gegen eine weitgehende rechtliche Gleichstellung wandten und durchsetzten, dass das Daueraufenthaltsrecht von der Erfüllung von Integrationsmaßnahmen, wie z.B. Sprachkursen, abhängig gemacht wurde (HAILBRONNER 2004). Erhalten blieb jedoch eine weitgehende, wenn auch nicht vollständige Gleichstellung von Drittstaatsangehörigen mit Daueraufenthaltserlaubnis mit Staatsbürgern im arbeits- und sozialrechtlichen Bereich sowie beim Zugang zum Bildungs- und Gesundheitssystem und zu Gütern und Dienstleistungen. Damit war der Spaltung des Arbeitsmarktes und der Diskriminierung aufgrund der Staatszugehörigkeit im Sozialbereich für langansässige Arbeitsmigranten, welche die österreichische Migrationspolitik lange Zeit geprägt hatte, ein Ende gesetzt. Es blieben allerdings die Übergangsfristen für den freien Arbeitsmarktzugang für Staatsangehörige der neuen Mitgliedstaaten.

Nachdem ursprünglich eine Adaption der österreichischen Gesetze durch entsprechende Novellen geplant war, zeigte sich schnell, dass dieser Systembruch eine Neukodifizierung der Rechtsmaterie verlangte. Das im Sommer 2005 gemeinsam mit dem neuen Asylrecht im österreichischen Parlament verabschiedete Gesetzespaket setzte die neuen EU-Regelungen im Wesentlichen um und brachte so auch Verbesserungen für langansässige Migranten, vor allem im aufenthaltsrechtlichen Bereich und beim Arbeitsmarktzugang.

Allerdings ist das Bestreben erkennbar, die EU-Regelungen möglichst restriktiv auszulegen. So wurden zum Beispiel die Rechte von Familienangehörigen nur äußerst eingeschränkt gewährt (KöNIG \& PerChinig 2005). Der im Fremdenrecht 1997 eingeführte absolute Ausweisungsschutz für im Land geborene und aufgewachsene Kinder und Jugendliche mit einem Drittstaatenpass wurde wieder aufgehoben; und der Zugang zum Daueraufenthaltsrecht, also zur „Niederlassungsbewilligung“ praktisch nur mehr für „Schlüsselkräfte“ und deren Familienangehörige ermöglicht.

\footnotetext{
6) KOM (2001) 127 endg

17) KOM (2001) 386 endg.
} 
Im Bereich der „Integrationsvereinbarung“ kam es zu einer Aufhebung der meisten Ausnahmen und zu einer Ausweitung der Kursbesuchspflicht von 100 auf 300 Stunden, wobei $50 \%$ der Kurskosten selbst zu tragen waren.

Insbesondere die Bestimmungen für den Familiennachzug wurden sehr restriktiv gefasst: Das Recht auf Familiennachzug wurde an die Nutzung der EU-Freizügigkeit geknüpft, was vor allem in Österreich lebende Bürger von Drittstaaten gegenüber Unionsbürgern beim Familiennachzug benachteiligt. Zudem wurden die nötigen Einkommensvoraussetzungen sehr hoch angesetzt (ECKER 2006), was zu einer Reihe von Verfahren vor den Höchstgerichten führte.

Während also, bedingt durch den Einfluss der EU und der Entscheidungen der Höchstgerichte, Ausländer mit Inländern am Arbeitsmarkt und im Sozialbereich zunehmend gleichgestellt wurden, setzte die „schwarz-blaue“ Koalition in den anderen migrationspolitischen Feldern restriktive Markierungen. Nachdem Österreich in den ersten Jahren seiner EU-Mitgliedschaft die EU-Politik mehr oder minder unbeteiligt erduldet hatte, wurde es nach den Erfahrungen mit den „Sanktionen“ zu einem wirklichen Akteur im Rat und konnte dort die eigenen Positionen durchsetzen. Anders als gegen Ende der 1990er Jahre, als europaweit ein Liberalisierungstrend zu beobachten war, gewannen nach 2001 überall in der EU restriktive Positionen an Gewicht. Damit gelangte auch die Position Österreichs, welche die nationale Souveränität im Bereich der Einwanderungs- und Integrationspolitik betonte, vom Rand in den "Main Stream" der EU-Politik.

\section{2 (Vorläufiger) Schluss: Migrations- und Integrationspolitik als Spiel auf mehreren Ebenen}

Während auf Bundesebene ein sicherheitspolitischer Fokus die Politikentwicklung prägte, änderte sich in den letzen zehn Jahren das Klima in den Bundesländern und Städten deutlich (Weigl 2009, S. 64). Die Stadt Wien, die bereits 1992 mit dem Wiener Integrationsfonds eine erste Integrationsstelle geschaffen hatte, allerdings in ihrer Migrationspolitik lange Zeit restriktiv blieb (JAWHARI 2000), begann unter der neu bestellten Integrationsstadträtin Renate BRAUNER (SPÖ) ab Beginn der 2000er Jahre mit einer engagierten Integrations- und Diversitätspolitik, die die Zuwanderung als Potenzial für die Stadtentwicklung sah und Wien als Einwanderungsstadt definierte (Antalovsky 2002). Auch in Vorarlberg entwickelte das Land eine neue Sichtweise auf das Thema und beauftragte den Verein „Okay-Zusammenleben“18) mit der Entwicklung einer regionalen Integrationsplattform (BURTSCHER 2009). Unterstützt durch das EUgeförderte Programm „Equal“, arbeiteten mehrere Städte in Vorarlberg, der Steiermark und in Niederösterreich Integrationsleitbilder aus (GÜNGÖR 2002; ZwICKLHUBER 2006) und machten Integration zu einem institutionell etablierten Politikfeld.

\footnotetext{
$\overline{{ }^{18)} \text { http://www.okay-line.at/ }}$
} 
Auch der im Jahr 2007 neu ins Amt gekommene Bundesinnenminister Günther PlatTER setzte - bei genereller Beibehaltung eines restriktiven Kurses - mit der Gründung der Integrationsplattform einen neuen Akzent (BUNDESMINISTERIUM FÜR INNERES 2008). Erstmals erkannte das Innenministerium Integration als politische Aufgabe an und setzte das Thema auf die Regierungsagenda. Zudem wurde, wenn auch primär symbolisch und unter Betonung der Dominanz der Regierungsinteressen, der Dialog mit der Zivilgesellschaft gesucht. Parallel dazu begann das Innenministerium, den in den 1960er Jahren vom UNHCR und der Republik gegründeten und vor allem in der Flüchtlingsintegration tätigen „Österreichischen Integrationsfonds“ als Kern einer neuen Integrationsbürokratie auszubauen. Er bekam nicht nur die Aufgabe der Koordination der Vergabe der Integrationskurse, sondern baute auch eine wissenschaftliche Abteilung aus und begann mit der Publikation von Informationsmaterial. ${ }^{19)}$ Mit der Wahl des Innenministers zum Landeshauptmann Tirols brachen die Aktivitäten der Integrationsplattform jedoch wieder weitgehend zusammen.

Seine Nachfolgerin Maria FEKTER nahm die Arbeit am „Nationalen Integrationsplan“ 2009 wieder auf, allerdings ohne die Nicht-Regierungsorganisationen einzubinden. Das von ihr präsentierte Grundlagenpapier (BUNDESMINISTERIUM FÜR INNERES 2009) stellte Migration vor allem als Bedrohung dar und betonte sicherheitspolitische Fragen, was nicht nur innerhalb der Nicht-Regierungsorganisationen, sondern auch bei den Bundesländern und Gemeinden auf Kritik stieß (ProfIL, 20.7.2009, S. 12).

In den Bundesländern war es inzwischen zu einer Neubewertung des Migrationsthemas gekommen. Mit der Verabschiedung von Integrationsleitbildern in Oberösterreich 2008 (GÜNGÖR 2008) und Tirol 2007 (GÜNGÖR 2006) und mit der Vorbereitung derartiger Leitbilder in anderen Bundesländern ${ }^{20)}$ hatte sich auf der Länderebene ein liberalerer Zugang entwickelt. Dazu trugen auch Änderungen der regionalen Herkunft und der sozialen Zusammensetzung der Migranten bei. Während zu Beginn der 2000er Jahre die Zuwanderung aus den ehemaligen „Anwerbeländern“, dem früheren Jugoslawien und der Türkei, zurückgegangen war, nahm sie aus den „,alten“, aber auch aus den „neuen“ EU-Ländern zu. Seit 2006 sind deutsche Staatsbürger - deren Einwanderung mit einer Anwerbeaktion im Rahmen der Zusammenarbeit der europäischen Arbeitsämter begann - die größte Einwanderungsgruppe in Österreich. Rund $60 \%$ aller Zuwanderer kamen im Jahr 2008 aus anderen EU-Staaten samt Deutschland. ${ }^{21)}$ Besonders die Neuzuwanderer aus dem EU-Raum, aber auch ein Großteil der Zuwanderer aus „Drittstaaten“, verfügen zumeist über mittlere und höhere Qualifikationen und entsprechen nicht mehr dem Klischee des „Gastarbeiters“.

Die geänderte geopolitische Situation und die Erweiterung der EU führten zudem zu einem Rückgang des nationalstaatlich steuerbaren Anteils der Zuwanderung. Die Zuwanderung aus der EU ist politisch nicht steuerbar, die Zuwanderung aus dem Nicht-EU-Raum betraf vor allem Asylwerber und den Familiennachzug, die sich beide ebenso weitgehend nationalstaatlicher Steuerung entziehen. Während auf Bundesebene

\footnotetext{
19) http://www.integration.at

${ }^{20)}$ Bis zum Abschluss dieses Manuskripts wurden in Niederösterreich, der Steiermark und in Vorarlberg Integrationsleitbilder erarbeitet. In Wien kam es 2009 zur Gründung einer Zuwanderungskommission.

${ }^{21)}$ Eigene Berechnungen basierend auf Daten von Statistik Austria. - http://www.statistik.at
} 
versucht wurde, durch Verschärfungen des Asyl- und Familiennachzugsrechts die Reste nationalstaatlicher Steuerfähigkeit zu sichern, setzte der demographische Wandel die Länder, Städte und Gemeinden unter Druck, Einwanderung als Realität zu akzeptieren und einen pragmatischen Umgang mit den Herausforderungen einer Einwanderungsgesellschaft zu entwickeln. In Wien lag im Jahr 2008 der Anteil von Migranten und Angehörigen der Zweiten Generation an der Gesamtbevölkerung bei mehr als einem Drittel. Die anderen großen Städte Österreichs erreichten Anteile um ein Viertel; und selbst in kleineren Städten und Gemeinden kamen oft 15\% der Bevölkerung aus dem Ausland (ÖsterReichISCHER INTEGRATIONSFONDS 2008, S. 19).

Während also die Bundespolitik die sicherheitspolitische Sichtweise forcierte, entstand in den Ländern und größeren Städten - oft mitinitiiert und beraten von Akteuren aus den Vorreiterländern Vorarlberg und Wien - eine Haltung, die stärker die Gleichstellung betont und Migration als Herausforderung und Entwicklungschance wahrnimmt. Damit steht die Politik der Länder heute der EU-Politik näher als dem Bund.

Anders als noch in den 1990er Jahren ist Migrationspolitik heute ein Spiel auf mehreren Ebenen: auf jener der Europäischen Union, der Mitgliedsstaaten, der Bundesländer und Gemeinden und der Sozialpartner, die jedoch wesentlich an Bedeutung verloren haben. Solche Verhältnisse machen radikale Paradigmenwechsel unwahrscheinlich und favorisieren schrittweise Politikentwicklung (MARKs 1993). Es ist daher auf mittlere Sicht zu erwarten, dass sich die Migrations- und Integrationspolitik auch in Österreich, ähnlich wie in anderen EU-Staaten, an einer Kombination aus restriktiver Steuerung der Neuzuwanderung und größerer Offenheit gegenüber kultureller Vielfalt orientieren wird, und dass sie sich zu einem, wenn schon nicht konsensualen, so doch weniger umstrittenen Politikfeld entwickelt.

\section{Literaturverzeichnis}

Antalovsky E. et al. (2002), Migration und Integration. Wien. - http:/www.europaforum.at/ downloads

Arendt H. (2007), Elemente und Ursprünge totalitärer Herrschaft. Antisemitismus, Imperialismus, totale Herrschaft. München.

B АUвӧск R. (1994), Transnational Citizenship. Membership and Rights in International Migration. Aldershot.

B Auвöск R. (2001), Gleichheit, Vielfalt und Zusammenhalt. In: Volf P., Bauвöck R. (Hrsg.), Wege zur Integration. Was man gegen Diskriminierung und Fremdenfeindlichkeit tun kann, S. 11-45. Klagenfurt/Celovec.

B Auвöck R. et al. (Hrsg.) (2006), Acquistion and loss of nationality. Policies and trends in 15 European states, 2, Country Studies. Amsterdam.

Bauböck R., Perchinig B. (2006a), Evaluation and Recommendations. In: Bauböck R. et al. (Hrsg.), Acquistion and loss of nationality. Policies and trends in 15 European states, 2, Country Studies, S. 431-479. Amsterdam.

Bauböck R., Perchinig B. (2006b), Migrations- und Integrationspolitik. In: Dachs H. et al. (Hrsg.), Politik in Österreich. Das Handbuch, S. 726-743. Wien. 
BAUMgaRTNER G. (2009), Verschwiegene Integration 1945-1961. Zur verdrängten Geschichte der größten Veränderung der österreichischen Bevölkerungsstruktur im 20. Jahrhundert. In: ÖGL, 3, S. 20-34.

Bendel P. (2007), Everything under Control? The European Union's Policies and Politics of Immigration. In: FAIST Th., EtTE A. (Hrsg.), The Europeanization of National Policies and Politics of Immigration. Between Autonomy and the European Union, S. 32-49. London.

BIFFL G. (2001), Mögliche Verdrängungsprozesse am Arbeitsmarkt. In: ÖsTERREICHISCHES INSTITUT FÜR WIRTSCHAFTSFORSCHUNG (Hrsg.), Arbeitsmarktrelevante Effekte der Ausländerintegration in Österreich, S. 279-300. Wien.

Bourdieu P., Coleman J.S. (Hrsg.) (1991), Social Theory for a Changing Society. Boulder.

BRATiC Lj. (2002), Diskurs und Ideologie des Rassismus im österreichischen Staat. Manus., Wien.

BundeSMINISTERIUM FÜR InNERES (Hrsg.) (2004), Asyl- und Fremdenstatistik 2004. Wien. - http:// www.bmi.gv.at/ downloadarea/ /Jahr2004.pdf (11.1.2006)

Bundesministerium FÜR InNERES (Hrsg.) (2008), Gemeinsam kommen wir zusammen. Expertenbeiträge zur Integration. Wien. - Im Downloadbereich von http://www.integration. $a t /$ abrufbar.

BUNDESMINISTERIUM FÜR InNERES (Hrsg.) (2009), Einführungspapier für den Nationalen Aktionsplan für Integration. Wien. - Im Downloadbereich von http://www.integration.at/ abrufbar.

BunZl J., HAFEZ F. (Hrsg.) (2009), Islamophobie in Österreich. Innsbruck.

Burtscher S. (2009), Zuwandern-Aufsteigen-Dazugehören. Etablierungsprozesse von Eingewanderten. Innsbruck.

Cicekli B. (1998), The Legal Position of Turkish Immigrants in the European Union. A Comparison of the Legal Reception and Status of Turkish Immigrants in Germany, the Netherlands and the UK. Ankara.

Cicekli B. (2004), Legal Integration of Turkish Immigrants under the Turkish-EU Association Law. Paper presented at the Conf. "Integration of Immigrants from Turkey in Austria, Germany and Holland", Bogacizi Univ., Centre for European Studies, February 27, 2004. - http://www.ces.boun.edu.tr/index2.html

Çinar D., W alrauch H. (2006), Austria. In: Bauböck R. et al. (Hrsg.), Acquistion and loss of nationality. Policies and trends in 15 European states, 2, Country Studies, S. 19-63. Amsterdam.

Council on Foreign Relations (Hrsg.) (2009), China's Internal Migrants. - http://www.cfr.org/ publication/12943/ (12.7.2009)

Die Presse, 17.3.2005.

ECKER J.M. (2006), Umsetzung der Rl 2003/86/EG betreffend das Recht auf Familienzusammenführung in Österreich. In: Migralex - Zeitschrift f. Fremden- u. Minderheitenrecht, 01, S. 13-19.

ECKER J.M. (2007), Umsetzung der R1 2003/86 durch das Fremdenrechtspaket 2005? In: Migralex - Zeitschrift f. Fremden- u. Minderheitenrecht, 02, S. 42-56.

EINEM C. (1990), Alternative Optionen der Zuwanderungspolitik. Manus (im Besitz des Verfassers). Wien.

Europäischer Gerichtshof für Menschenrechte (Hrsg.) (1996), Gaygusuz gegen Österreich. Urteil vom 16. September 1996, NL 1996, S. 135 (NL 96/5/8).

EUROPÄISCHER RAT VON TAMPERE, 15. und 16. Oktober 1999, Schlussfolgerungen des Vorsitzes. - http://www.europarl.europa/eu/summits/tam_de.htm (24.4.2005)

FAIST Th. (2006), The Migration-Security Nexus: International Migration and Security before and after 9/11. In: Bodemann M., Yurdakul G. (Hrsg.), Migration, Citizenship, Ethnos. Incorporation Regimes in Germany, Western Europe and North America, S. 157-174. London. 
Fassmann H., Münz R. (1995), Einwanderungsland Österreich? Historische Migrationsmuster, aktuelle Trends und politische Maßnahmen. Wien.

Gächter Au. (1995), Integration und Migration. In: SWS-Rundschau, 35, 4, S. 435-438.

GÜNGÖR K. (2002), Integrationsleitbild der Stadt Dornbirn, mit integriertem Maßnahmenplan. Manus. Dornbirn - Basel.

GÜNGÖR K. (2006), Integrationskonzept des Landes Tirol mit Maßnahmenempfehlungen. Innsbruck.

GÜNGÖR K. (2008), Einbeziehen statt Einordnen. Zusammenleben in Oberösterreich, Integrationsleitbild des Landes Oberösterreich. Linz.

Hailbronner K. (2004), Die Richtlinie zur Familienzusammenführung und zum langfristigen Aufenthalt von Drittstaatsangehörigen - Entstehungsgeschichte und aktuelle Fragen der Auslegung. Vortrag, Fachtagung zur EU-Immigrations- und Integrationspolitik, Arbeiterkammer Wien 19.2.2004. Manus.

Hammar T. (1990), Democracy and the Nation State. Aliens, Denizens and Citizens in a World of International Migration. Aldershot.

HunN K. (2004), „Nächstes Jahr kehren wir zurück ...“. Die Geschichte der türkischen Gastarbeiter in der Bundesrepublik. Freiburg.

ICDuYGu A. (2006), Gaining from Migration. A Comparative Analysis and Perspective on how Sending and Receiving Countries can gain from Migration. Case Study on Turkey. Paris.

JAWHARI R. (2000), Wegen Überfremdung abgelehnt. Ausländerintegration und symbolische Politik. Wien.

Juen G., Perchinig B., Volf P.-P. (1998), Migrationspolitik: Zur Europäisierung eines Gastarbeitermodells. In: TÁlos E., Falkner G. (Hrsg.), EU-Mitglied Österreich. Gegenwart und Perspektiven: Eine Zwischenbilanz, S. 201-221. Wien.

Karner St., Stangler G. (2005), Österreich ist frei. Der Österreichische Staatsvertrag 1955. Beitragsband Schallaburg 2005. Horn - Wien.

Koller J. (1998), Kommunale Integrationspolitik. Eine Analyse des Wiener Integrationsfonds im Kontext sozialdemokratischer Interessenspolitik. Wien, Univ. Wien, Dipl.-Arb.

König K., Perchinig B. (2005), Austria. In: Niessen J., Schibel Y., Thompson C. (Hrsg.), Current Immigration Debates in Europe. A Publication of the European Migration Dialogue, S. 13-47. Brussels.

König K., Stadler B . (2003), Entwicklungstendenzen im öffentlich-rechtlichen und demokratiepolitischen Bereich. In: Fassmann H., Stacher I. (Hrsg.), Österreichischer Migrationsund Integrationsbericht, S. 226-261. Wien.

Kraler A., Stepien A. (2006), Immigrant and Immigration Policy Making in Austria. Manus. Wien. Ludwig M. et al. (Hrsg.) (1995), Der Oktoberstreik 1950. Wien.

Marks G. (1993), Structural Policy and Multi-Level Governance in the EC. In: Cafruny A.W., Rosenthal G. (Hrsg.), The State of the European Community, 2, The Maastricht Debates and Beyond, S. 397-411. Boulder.

Marshall T.H. (1992), Citizenship and Social Class. In: Marshall T.H., Bottomore T. (Hrsg.), Citizenship and Social Class, 2. Aufl. London.

MatuscheK H. (1986), Ausländerpolitik in Österreich 1962-1985. Der Kampf um und gegen die ausländische Arbeitskraft. In: Journal f. Sozialforschung, 25, 2.

MüNZ R., Zuser P., KytiR J. (2003), Grenzüberschreitende Wanderungen und ausländische Wohnbevölkerung: Struktur und Entwicklung. In: Fassmann H., Stacher I. (Hrsg.), Österreichischer Migrations- und Integrationsbericht, S. 20-62. Klagenfurt/Celovec.

ÖSTERREICHISCHER InTEGRATIONSFONDS (Hrsg.) (2008), Integration. Zahlen, Daten, Fakten 2008. Wien.

Perchinig B. (2006a), Die EU-Migrationspolitik und die migrationspolitische Entwicklung in Österreich. In: Fassmann H. (Hrsg.), Zweiter Österreichischer Migrations- und Integrationsbericht, S. 131-145. Klagenfurt/Celovec. 
Perchinig B. (2006b), Einwanderungs- und Integrationspolitik. In: TÁlos E. (Hrsg.), SchwarzBlau. Eine Bilanz des „Neu-Regierens“, S. 295-312. Wien - Münster.

Perchinig B. (2009), Von der Fremdarbeit zur Integration? (Arbeits)migrations- und Integrationspolitik in der Zweiten Republik. In: ÖGL, 3, S. 1-19.

Perchinig B. (im Druck), Between federal republicanism and communitarian provincial identity. Citizenship testing in Austria. In: Ersboll E., Kostakopoulou D., van Oers R. (Hrsg.), Language and Integration Tests for Newcomers and Future Citizens, S. 25-50. Leiden - Boston, Brill.

Profil, 20.7.2009.

RAAB J. (1961), Die Kammerorganisation der Gewerblichen Wirtschaft. Ansprache am Kammertag der Bundeskammer anlässlich der Wahl des Präsidenten, 26. Mai 1961. - http:// wko.at/mk/60jahre/Raab_26_05_1961.pdf

Rohsmann K. (2006), Die „Integrationsvereinbarung“ der Fremdengesetznovelle 2002. Integrationsförderung durch Sprach(kurs)zwang? Wien, Univ. Wien, Dipl.-Arb.

Sensenig E. (1998), Reichsfremde, Staatsfremde und Drittausländer. Immigration und Einwanderungspolitik in Österreich. Forschungsbericht. Salzburg, Ludwig-Boltzmann-Inst. f. Gesellschafts- u. Kulturgeschichte.

Sosysal Y.N. (1994), Limits of Citizenship. Migrants and Postnational Membership in Europe. Chicago.

Stieber G. (1995), Volksdeutsche und Displaced Persons. In: Heiss G., Rathкolb O. (Hrsg.), Asylland wider Willen. Flüchtlinge in Österreich im europäischen Kontext seit 1914, S. 140-156. Wien.

TÁLOs E. (2006), Sozialpartnerschaft. Austrokorporatismus am Ende? In: DACHS H. et al. (Hrsg.), Politik in Österreich. Das Handbuch, S. 425-443. Wien.

Torpey J. (2000), The Invention of the Passport. Surveillance, Citizenship and the State. Cambridge.

United Nations (Hrsg.) (2008), International Migrant Stock: The 2008 Revision. - http://esa. un.org/migration/p2kOdata.asp (12.7.2009)

United Nations, Department of Economic and Social Affairs, Statistics Division (Hrsg.) (1998), Recommendations on Statistics of International Migration (= Statistical Papers Series M, 58, 1). New York.

Volf P.-P. (1995), Der politische Flüchtling als Symbol der Zweiten Republik. In: Zeitgeschichte, $11-12$, S. 415-436.

Weigl A. (2009), Migration und Integration. Eine widersprüchliche Geschichte. Wien.

Wimmer H. (1985), Zur Ausländerbeschäftigungspolitik in Österreich. In: Wimmer H. (Hrsg.), Ausländische Arbeitskräfte in Österreich, S. 5-33. Frankfurt am Main.

WOLLNER E. (1996), Auf dem Weg zur sozialpartnerschaftlich regulierten Ausländerbeschäftigung in Österreich. Die Reform der Ausländerbeschäftigung und Anwerbung bis Ende der 1960er Jahre. Wien, Univ. Wien, Dipl.-Arb.

Zolberg A. (1991), Bounded States in a Global Market: The Uses of International Labor Migrations. In: Bourdieu P., Coleman J.S. (Hrsg.), Social Theory for a Changing Society, S. 301-335. Boulder.

Zwicklhuber M. (2006), Integrationspolitik auf Länderebene. Eine Bestandaufnahme in Niederösterreich. Krems, Donau Univ. Krems, Master-These. 